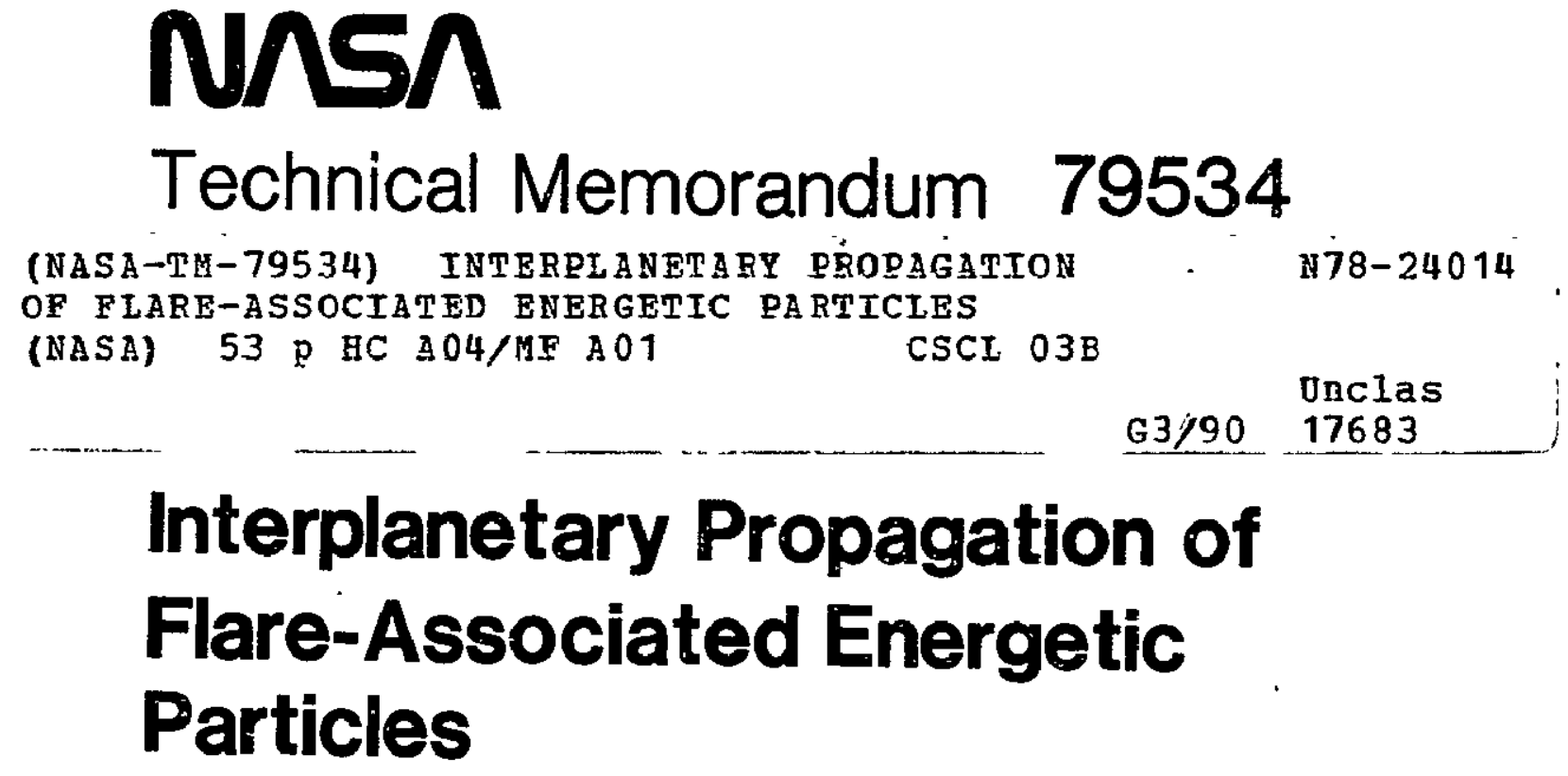

Linda S. Ma Sung and James A. Earl

April 1978

National Aeronautics and

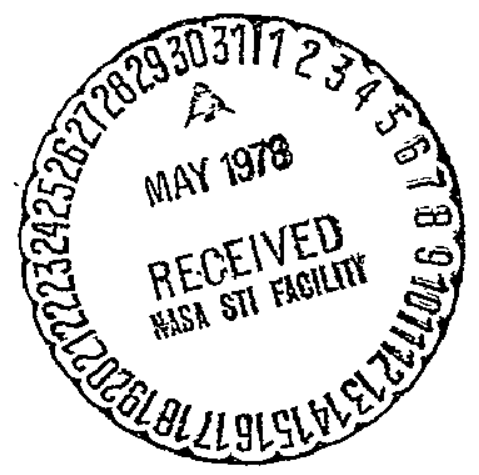

Space Administration

Goddard Space Flight Center

Greenbelt, Maryland 20771 


\title{
INTERPLANETARY PROPAGATION OF FLARE-ASSOCIATED \\ ENERGETIC PARTICLES \\ by
}

\author{
Linda S. Ma Sung* and James A. Ear1 \\ Department of Physics and Astronomy \\ University of Maryland \\ College Park, Maryland 20742
}

*Also Laboratory for High Energy Astrophysics, NASA/Goddard Space Flight Center, Greenbelt, MD 20771

To be published in the June 1978 issue of the Astrophysical Journal 
ABSTRACT

A propagation model which combines, a Gaussian profile for particle release from the sun, with interplanetary particle densities predicted by focused diffusion, is proposed to explain the propagation history of flare-associated energetic particles. This model, which depends on only two parameters, successfully describes the time-intensity profiles of 30 proton and electron events originating from the western hemisphere of the sun. On comparing the predicted and observed density profiles, it is found that the scattering mean free path $\lambda$ is on the order of 0.1 to $0.3 \mathrm{AU}$ for $4-80 \mathrm{MeV}$ protons and is a factor of 2 to 3 smallex for 0.5-1.1 MeV and 3-12 MeV electrons. Since the rigidities here differ by more than two orders of magnitude, this implies that $\lambda$ is only weskiy dependent on rigidity. The rigidity dependence of $\lambda$, as well as its magnitude, strongly disagrees with that predicted by the quasilinear theory of pitch-angle scattering. Generally, particles are released from the sun. over a finite interval. Approximating this profile of injection by a truncated Gaussian, the r.m.s. width $\sigma$ is less than 1 hour for most events. The injection width $\sigma$ decreases with velocity, but it does not depend on rigidity. In almost all events, particle release begins at the time of flare acceleration.

Subject headings: cosmic rays: general - hydromagnetics interplanetary medium - sun: flares 


\section{INTRODUCTION}

A1though the behavior of charged particles in interplanetary space has been studied for more than two decades, the exact nature of their propagation is not yet clearly understood. Classically, the scattering of charged particles in random magnetic fields was treated in terms of diffusion (Parker 1956, 1963; Meyer, Parker, and Simpson 1956; Bryant et al. 1962; Jokipii 1966, 1967; Burlaga 1967; Roelof 1966; 1968; Englade 1971; Lupton and Stone 1973; Webb et al. 1973; Webb and Quenby 1973). Recent work on solar particle events has reached a consensus that the scattering mean free path of low energy protons is on the order of 0.1-0.2 AU (Ianzerotti et al. 1973; Roelof and Krimigis 1973; Reinhard and Wibberenz 1974; Ma Sung, Van Hollebeke, and McDonald 1975; Countee and Lanzerotti 1976; Zwickl 1976; Hamiltton 1977; Ma Sung 1977), which is an order of magnitude larger than the mean free path estimated from the intexplanetary magnetic power spectrum by Jokipii and Coleman (1968). In the regime of weak scattering, implied by this large mean free path, 'the treatment of particle propagation in terms of simple diffusion is not appropriate. Rēcently, (Earl 1976a,b) and Earl and Bieber (1977) developeda theory which treats particle transport in this regime, and which gives a quantitative prediction of both the intensity and the anisotropy of the particle fluxes in space based on a Fokker-P1anck description of pitch-angle scattering (Jokipii 1966, 1967; Koel of 1968). This theory a1so-included the focusing effect which results from conservation of the first adiabatic invariant for particles propagating along a diverging magnetic field (Roelof 1966). In this paper, the basic propagation process of flare-associated particles in interplanetary space is studied in terms of a detailed model which incorporates a coronal injection profile and the theory of focused diffusion in interplanetary space. This model assumes purely longitudinal propagation uncomplicated by azimuthal transport in the corona or interplanetary space. 
Unlike previous works on diffusion which involked an escaping boundary beyond 1. AU to account for the observed exponential decay of particle events, this model treats interplanetary propagation in terms of only one parameter, (V/AL), which gives the relative strength of scattering to focusing, and which predicts an exponential decay in agreement with observations. Because the model takes into account non-diffusive effects which are particularly significant during the initial phase of an event, we have been able to deduce, by comparing the observed onset and decay phases with the model, not only the properties of interplanetary propagation, but also the characteristics of coronal escape. While the existence of a finite coronal injection has been pointed out previously (Feit 1973; Nielsen, Pomerantz, and West 1975; Palmer, Palmeira, and Allum 1975; Reinhard 1975; Ng and Gleeson 1976; Wibberenz, Lanzerotti, and Venkatesan 1976), it is firmly established here for 30 events, and the injection time is demonstrated to depend on particle velocity but not on rigidity.

Particle data were collected by the Goddard Space Flight Center cosmic ray experiments on IMPS IV and V. Some thirty events associated with flares on the western hemisphere of the sun were tested against the mode1.

Section II describes the particle data and flare association. Section. III. gives the propagation model. In section IV, the profiles predicted by the model. are compared with electron and proton events, and in section $V$, the results are. discussed. This study gives important new information on a) the rigidity, and. velocity dependence of low enexgy particle propagation in the intexplanetaxy magnetic field, b) the velocity dependence of particle release from the sun, c) the time of release of particles to flare acceleration. 
II. DATA

Electron and proton data were obtained by the Goddard Space Flight Center cosmic ray experiments aboard the highly eccentric earth orbiting satellites IMPs IV and V. They covex five years between May 1967 and December 1972 with short interruptions from May 1969 to June 1969 and from November 1971 to February 1972.

The GSFC instrument consisted of two $\mathrm{dE} / \mathrm{dx}$ vs. E. telescopes. A low energy detector (LED) detected protons and aipha particles between 4 and 20 MeV/nucleon, and a medium energy detector (MED), detected protons and alpha particles between 20 and $80 \mathrm{MeV}$ and electrons between 3 and $12 \mathrm{MeV}$. The thin $\mathrm{dE} / \mathrm{dx}$ element, MED A, was used to detect electrons in the 0.5-1.1 MeV energy range. This element had a geometric factor of $60 \mathrm{~cm}^{2}$-ster. The axis of the MED was parall to the spacecraft spin axis which was normal to the ecliptic plane; the axis of the LED was normal to the spin axis with a field of view of $<20^{\circ}$. The satellite spin rate was approximately $23 \mathrm{rpm}$. These detector systems were described in detail by Bryant et al. (1962), Kinsey (1970), and Van Hollebeke, Wang, and McDonald (1974). Detectors on IMP IV were essentially identical to those on IMP $V$.

The theory described in Section III predicts not only the isotropic particle density, but also the detailed form of the anisotropy. Obviously, it would be very desirable to compare these predictions with observed anisotropies. Unfortunately, the present data define only the isotropic component, for they were derived from telescope counting rates averaged over spin azimuth.

During the five-year period, 185 solar cosmic ray events were detected. Their time histories were catalogued by Van Hollebeke et al. (1974). To obtain a reasonable resolution above the galactic cosmic ray background and to provide a better separation from co-rotating events, the proton flux above $20 \mathrm{MeV}$ was required to exceed $10^{-4}$ particles $/ \mathrm{cm}^{2}-\mathrm{sec}-\mathrm{sr}-\mathrm{MeV}$ for several hours. Among the 185 events detected, 125 were associated with an optical flare, a soft $X$-ray $(2-20 \AA)$ burst, spectral type II, III, IV radio 
bursts or intense microwave bursts with peak flux $\gtrsim 500 \times 10^{-22}$ watts $\mathrm{m}^{-2} \mathrm{~Hz}^{-1}$ àt frequencies above $3000 \mathrm{MHz}$ (Van Hoilebeke, Ma Sung, and McDonald 1975).

In this paper, we consider 70 proton and electron events, which origiginated between $\backsim 20^{\circ}$ and $w 100^{\circ}$, and which comprised approximately $56 \%$ of all identified events. The longitudes span a region of fast propagation and preferred connection (Reinhard and Wibberenz 1974; Van Hollebeke et a1. 1975; Ma Sung et a1. 1975), within which coronal effects on particle propagation are minimal. To properly compare the particle time histories with model prediction, the events selected for analysis were required to have a sufficiently clean background (i.e. no significant flux of solar cosmic rays from a previous event) and to have a maximum proton intensity above $4 \times 10^{-4} / \mathrm{cm}^{2}-\mathrm{sec}-\mathrm{sr}-\mathrm{MeV}$ at $40 \mathrm{MeV}$. In addition, events which showed distinct local perturbations or which occurred at the time of perigee passage were excluded. These requirements reduced the sample to a total of 31 events, of which 30 were found to agree with the model described in Section III. AII but 3 events, those on January 28, 1970, January 29, 1970, and April 22, 1971, were well associated not only with $\mathrm{H}_{\alpha}$ and $\mathrm{X}$-ray emissions, but also with type II, type IV or intense microwave bursts. Therefore, we believe that the flare association is sound in all except perhaps these 3 cases. 
IIT. THE INTERPLANTARY PROPAGATION MODEL

The model is based on the theory of focused diffusion (Earl 1976a), which describes the particle distribution function $f(\dot{\mu}, z, t)$ in terms of solutions of the one dimensional Fokker-Planck equation,

$$
\frac{\partial f}{\partial t}+\mu V \frac{\partial f}{\partial z}=\frac{1}{2} \frac{\partial}{\partial \mu} \phi \frac{\partial f}{\partial \mu}-\frac{V}{2 L}\left(1-\mu^{2}\right) \frac{\partial f}{\partial \mu}
$$

where $\mu$ is the cosine of the pitch angle; $z$ is the distance along the guiding field, $V$ is the particle velocity, and $L$ is the scale length for spatial variation of the guiding field, $L=-B /(\partial B / \partial z)$. The Fokkex-Planck coefficient for pitch-angle scattering was assumed to have the form,

$$
\phi(\mu)=A|\mu|^{q-1}\left(I-\mu^{2}\right)
$$

According to quasilinear theory (Jokipii 1966, 1971), the parameter $q$ is the spectral index for intexplanetary field fluctuations, and the parameter A depends on the velocity and the rigidity of the particles, and on the amplitude of the random fields. Because the validity of quasilinear theory has been questioned in recent years, it is important to realize that this form was adopted, in the absence of a definitive analysis of pitch-angle scattering, as a working formula whose parameters conveniently characterize the intensity $A$ and the anisotropy. $q$ of the scattering. According to this point of view, the magnitude and rigidity. dependence of these parameters will be regarded here as empirical values to be determined experimentally rather than as calculated values related to the interplanetary fluctuation spectrum by quasilinear theory. In this way, we attempt to specify the 
dependences that must be predicted by sound theories of pitch-angle scattering.

The assumption of one dimensional propagation along the mean field is supported by the observation of strongly field aligned anisotropies during the initial phase of low energy proton and electron events (McCracken, Rao, and Bukata 1967; McCracken, Rao, and Ness 1968; Fan et aI. 1968; Allum et al. 1971; Rao et al. 1971; Roelof and Krimigis 1973). Other evidence for field aligned propagation includes the distribution of events in heliolongitude which shows a pronounced peak between $w 30^{\circ}$ and $w 60^{\circ}$ (Van Hollebeke et al. 1975), and the distribution of the event risetimes which shows a broad minimum between $W 20^{\circ}$ and $w 90^{\circ}$ (Barouch, Gros and Masse 1971; Datlowe 1971; Simnett 1971; McKibben 1972; Lanzerotti 1973; Reinhard and Wibberenz 1974; Ma Sung et a1. 1975; Van Hollebeke et a1. 1975; Ma Sung 1977).

Except for trivial special cases, exact solutions of the transport equation cannot be obtained in closed form. Consequently, to obtain. useful approximate solutions, Ear1 (1976a) expanded the distribution function in terms of the eigenfunctions of an operator that describes the combined effect of scattering and focusing. This representation leads to a matrix fomulation that is completely equivalent to the original transport equation. However, this formulation in terms of infinite matrices can be drastically truncated with no appreciable loss in accuracy, because only the first few terms of the eigenfunction series are significant. Thus, the present model is based upon analytical solutions which retain only the two lowest order eigenfunctions, However, the phenomenon of dispersion, which involves two eigenfunctions of the next higher order, was àlso taken into account approximately by the convolution method described below. 
It seems best not to repeat here formulae for the analytic solutions, for they are complicated expressions that involve many parameters. Instead, we emphasize the crucial point that the exact form of these solutions is completely specified by only two parameters, $q$ and (V/AL). The latter parameter, which is the ratio of the scattering length (V/A) to the focusing length $\mathrm{L}$, chaxacterizes the importance of focusing relative to scattering. Throughout this work, a fixed value, $q=1.5$, was adopted. This value approximates the observed spectral index of interplanetary magnetic fluctuations. Similarly, the focusing length $L$ was fixed at its value at $1,2 \mathrm{AU}, \mathrm{L}=\mathrm{r} / 2=0.6 \mathrm{AU}$. Thus, in effect, the only free parameter was the scattering iength (V/A).

The parameter (V/A) and the index $q$ are related to the diffusion coefficient K by (Earl 1974b)

$$
K=\frac{\left(V^{2} / A\right)}{(2+q)(4-q)}
$$

Because $K=(1 / 3) V \lambda$, where $\lambda$ is the mean free path, it follows that,

$$
\lambda=\frac{3}{(2-q)(4-q)}\left(\frac{V}{A}\right) .
$$

Hence, $\lambda$ increases as (V/A) increases while $q$ is constant, or as $q$ increases while (V/A) is constant. During typical western hemisphere events, in which $(\mathrm{V} / \mathrm{AL}) \approx 0.1-0.2$ as obtained in this analysis, the diffusion coefficient and the mean free path for $40 \mathrm{MeV}$ protons $(\mathrm{V}=1.98 \mathrm{AU} / \mathrm{hr})$ range from $(0.6$ to 1.2$) \times 10^{22} \mathrm{~cm}^{2} / \mathrm{sec}$ and from 0.14 to $0.28 \mathrm{AU}$, respectively. The theoretical density profile we compared with experimental data is the function $\mathrm{F}_{0}{ }^{+}\{z, t\}$, whose specific form, given by $\operatorname{Earl}$ (1976a, eq. 55), 
will not be repeated here for the reason given above. It was evaluated by substituting in Earl's equation (55) the parameters defined by his equations (37) to (41) which were calculated as a function of (V/A) from the numbers that appear in his Table $I$ for $q=1.5$. The profile. $F_{0}^{4}\{z, t\}$ consists of a $\delta$-function coherent pulse, which moves with speed $v_{+} \simeq 0.6 \mathrm{~V}$ outward aIong the guiding fieId, and a wake, which trails behind the pulse. A second component of the general solution, the function $F_{0}{ }^{-}\{z, t\}$, which could be significant if particles were injected inward, was not included because flare injection clearly corresponds to the outward injection described by $\mathrm{F}_{0}^{+}\{\mathrm{z}, \mathrm{t}\}$.

In a magnetic field of the form $B \propto 1 / r^{2}$, the focusing length $L$ is not constant, for it varies as $L=r / 2$. Thus, as $L$ increases with distance from the sun, the focusing parameter (V/A) decreases from the supercoherent regime $(V / A) \gg 1$, within which solar particles propagate in a well collimated beam, to the regime of focused diffusion V/AL $\approx .1$, within which the present formulation applies. Because this transition from supercoherent to diffusive propagation occurs when $(V / A L)=V /(A r / 2) \simeq I$, or when $r \simeq 2(V / A)$, we assumed that the injection of particles occurred at $z=2(\mathrm{~V} / \mathrm{A})$, rather than at $z=0$, and at time $t=z / V_{+}=\left(\frac{2}{V_{+}}\right)\left(\frac{V}{A}\right)$, which is the time it takes the supercoherent pulse to reach the transition point at speed $V_{+}$.

The function $\mathrm{F}_{0}^{+}\{z, \dot{t}\}$ was obtained by including only the first two terms in the eigenfunction series that represents the particle distribution function. If the next two higher order terms are included, the $\vec{\delta}$-function pulses in the density assume a finite spread due to dispersion. Physically, dispersion describes statistical fluctuations within a bunch of particles whose velocities wander randomly in direction over one henisphere with an 
average speed along the field of approximately $0.6 \mathrm{~V}$. An approximate solution of this more rigorous treatment, valid when (V/AL) $\lesssim 1$, is obtained by convolving $\mathrm{F}_{0}{ }^{+}\{z, t\}$ with a coherent Gaussian (Earl 1976b),

$$
F_{0}\left\{z^{\prime}, t^{\prime}\right\}=\int_{-\infty}^{\infty} d z_{0} \frac{\exp \left\{-\left(z-z_{0}\right)^{2} / 4 D_{*} t\right\}}{2\left(\pi D_{*} t\right)^{\frac{1}{2}}} \cdot F_{0}{ }^{+}\left\{z_{0}, t\right\},
$$

where $D_{\star}=0.0405\left(V^{2} / A\right)$ is the coefficient of dispersion introduced by Earl (1974a, eq. 83) and discussed by Kunstmann and AIpers (1977), and where $z^{\prime}=z+2(V / A), t^{\prime}=t+\left(\frac{2}{V_{+}}\right)\left(\frac{V}{A}\right)$.

Figure 1 shows as solid lines the density profiles for $40 \mathrm{MeV}$ protons predicted by equation (5) for $(\mathrm{V} / \mathrm{AL})=0.2$ and 0.7 . To illustrate the importance of the coherent effect, the dashed lines show separately the Gaussian profiles into which the coherent $\delta$-functions are converted by the convolution operation. At the right, where the choice $(\mathrm{V} / \mathrm{AL})=0.7$ leads to virtually coherent or scatter-free propagation, the coherent Gaussian is a dominant feature which overshadows the inconspicous tail that arises from the diffusive wake. But, even at the left, where the choice $(\mathrm{V} / \mathrm{AL})=0.2$ leads to relatively "diffusive pröpagation dominated ' by the wake, the peak intensity provided by the coherent Gaussian amounts to a substantial fraction (36\%) of the total intensity present, at' the time of maximum. To test the accuracy of ; the . approximations leading to equation (5), John. Bieber (1977) càlculated density profiles which apply to exactly the same conditions assumed in Figure 1, but took rigorously into account all of the first four eigenfunctions. Evidently, the differences between the rigorous calculation, whose predictions appear as dotted lines in Figure 1 , and the approximate treatment invoked here are insignificant. 
Equation (5) gives the particle density following an impulsive injection at the sun. On comparing the temporal profiles of density calculated at $z^{\prime}=1.2$ AU with the tempōräl prófillès of western flare events, we found that, in almost all cases, the calculated density successfully fits the events during the decay phase, but the initial rise to maximum is consistently shorter than that observed. Although perpendicular diffusion might cause this discrepancy, this possibility is considered unlikely because it would imply that the mean propagation time $\tau$ has a form (Jokipii and Parker 1968, 1969)

$$
\tau \propto \frac{1}{\mathrm{~K}} \approx \frac{1}{\mathrm{~B}}
$$

that is not obseved.

A second, more viable, possibility is that the additional delay is due to a coronal effect which makes the release of particles from the sun persist over a finite time interval. (Feit 1973; Nielsen et al.

1975; Palmer et al. 1975; Reinhard 1975; Ng and ĠIeeson 1976;

Wibberenz et a1. 1976). Because equation: (5) refers to an injection whose temporal profile can be represented by a $\delta$-function, the solution for an injection $f(t)$ of finite duration is the convolution of $f(t)$ with this Green's function. As an approximation, it was assumed that the injection profile is a Gaussian with a standard deviation $\sigma$. The peak of the Gaussian was placed at $t=3 \sigma$ and the Gaussian was truncated in such a way that particles are not released before $t=0$. The convolution of the Gaussian with equation (5) is:

$$
\left.F\left\{z^{\prime}, t\right\}=\int_{0}^{t} d t^{\prime} F_{0}\left\{z^{\prime}, t^{\prime}\right\} \frac{\exp \left(-\frac{\left(t-t^{\prime}-3 \sigma\right)^{2}}{2 \sigma^{2}}\right\}}{(2 \pi)^{\frac{1}{2}} \sigma}\right] \text {, }
$$

where $t$ is the time of observation. The lower limit of this integral is zero, because $F_{0}\left\{z^{\prime}, t^{\prime}\right\}=0$ for $t^{\prime} \leq 0$. The upper limit is $t$, because the 
Gaussian was truncated for $t-t^{\prime} \leq 0$. Clearly, this is not necessarily the only possible solution which can describe a particle event. However, it will become evident that, for most events, this injection profile does give a satisfactory representation of the initial phase. 


\section{MODEL FITTING}

The entire time-intensity profiles of thirty events were fitted by. eye using thi.s model. To demonstrate the fitting of a typical event, such as the one that occurred on July 7,1970 , we show in figure 2 the 0.5-1.1 MeV electron and 30-50 MeV proton time-intensity profiles compared with densities calculated from equation (6). Solid curves give profiles for the values of (V/AL) which best fit the observed particle density during the decay phase. Dashed curves give profiles for two neighboring values of (V/AL). After the most appropriate value of (V/AL) was found by comparing the calculated and observed decay phases, the Gaussian injection width $\sigma$ was varied in steps of 0.2 hours until a best fit over the entire profile was found. For the event in Figure 2, this procedure leads to the following estimates of (V/AL) and $\sigma$ for electron and $\backsim 40 \mathrm{MeV}$ protons:

$$
\begin{array}{rlrl}
(V / A L) & =0.06 \pm 0.005, & \\
\sigma & =0.4 \pm 0.1 \text { hours } & & 0.5-1.1 \mathrm{MeV} \mathrm{e} \\
(\mathrm{V} / \mathrm{AL}) & =0.14 \pm 0.01, & & \\
\sigma & =0.6 \pm 0.1 \text { hours. } & &
\end{array}
$$

In figure 2, note that the rigidity of the electrons is $2 \frac{1}{2}$ orders of magnitude smaller than that of the protons, but that the scattering length of the electrons is only a factor or 2 smaller than that of $40 \mathrm{MeV}$ protons. This weakness of rigidity dependence in interplanetary propagation has been found in many events. A similar conclusion was drawn by Countee and Lanzerotti (1976). As will be discussed later, the rigidity dependence of the coronal injection profile is even weaker.

Figure 3 shows a sample of 0.5-1.1 MeV electron events. Figure 4 shows a corresponding sample of $40 \mathrm{MeV}$ proton events. These events cover the entire range of (V/AL) from coherent propagation to diffusion. In each case, 
to allow proper study of the behavior near onset, the background flux was subtracted. For purposes of comparison, the density calculated for the indicated parameters was averaged over the same time interval as the data. Figures 3 a and 4 a show the time histories for an event on December 29, $1967\left(W 77^{\circ}\right)$ which had the extremely sharp time profile of scatter-free microevents. Because this event was very brief, electron data were sampled every three minutes, and proton data were averaged over fjfteen-minute intervals. In this case, the best fit was obtained for $\sigma=0$ which corresponds to impulsive injection. The ratios $(\mathrm{V} / \mathrm{AL})=0.48$ for $0.5-1.1 \mathrm{MeV}$ electrons, and $(\mathrm{V} / \mathrm{AL})=0.85$ for $40 \mathrm{MeV}$ protons obtained for this event are the highest of all events analyzed. These large values of (V/AL) correspona to highly coherent propagation. However, note that the propagation. of protons was. more coherent than that of electrons. Figures $3 \mathrm{~b}$ and $4 \mathrm{~b}$ show a microevent on August 3, $1967\left(685^{\circ}\right)$. during which electron and proton data were averaged over fifteen minutes and thixty minutes, respectively. Essentially the same injection width, $\sigma: \sim 0.4 \cdot \mathrm{hr}$, descxibes the initial phase of both electrons and $40 \mathrm{MeV}$ protons.

The remaining events in Figures 3 and 4 become progressively more diffusive as (V/AL) decreases. These include the 0.5-1.1 MeV electron and $\checkmark 40 \mathrm{MeV}$ proton events on August 14, $1968($ (W80 $)$; February 24, $1969\left(\right.$ (W32 $\left.2^{\circ}\right)$; February 25, $1969\left(\mathrm{~W} 37^{\circ}\right)$; Apri1 6, $1971\left(W 80^{\circ}\right)$; November 24, $1969\left(W 31^{\circ}\right)$; and March 27, $1969\left(\mathrm{W6} 8^{\circ}\right)$. Figure $3 \mathrm{~g}$ shows, in addition, the $3-12 \mathrm{MeV}$ electron.event on February 25, 1969 (W37 ${ }^{\circ}$ ). This event was among the very few for which 3-12.MeV electron flux could be analyzed. In general, the electron flux at 3-12 MeV was masked by a combination of a high electron background and a steep electron spectrum. By comparing the electron time histories in the interval of $0.5-1.1 \mathrm{MeV}$, and $3-12 \mathrm{MeV}$ (Figures $3 \mathrm{~d}$ and $3 \mathrm{~g}$ ), we note that electrons have essentially the same injection profile and 
propagate in about the same manner over the velocity range $\beta=0.9-1.0$ and rigidity range $R=I-8 \mathrm{MV}$. The corresponding $40 \mathrm{MeV}$ protons (the second event in Figure 4 d) are characterized by a value of (V/AL) which is only a factor of 2,5 larger.

For the event of April 6, $1971\left(\right.$ W8 $\left.0^{\circ}\right)$, which is a typical western hemisphere event, Figure 5 compares the propagation characteristics of protons over the range from 4 to $80 \mathrm{MeV}$, corresponding to a range of rigidities $\mathrm{R}$ from 87 to $400 \mathrm{MV}$, and a range of velocities $\beta$ from 0.09 to 0.39. Figure 3 e shows the temporal profile of the 0.5-1.1 MeV electrons for this event. Solid curves give the best estimates of (V/AL) in each range, while dashed curves show possible fits in steps of 0.02 about the best estimates of (V/AL). Here (V/AL) increases with decreasing proton energy. This means that propagation of lower energy protons is more coherent than that of higher energy ones. Since the mean free path is proportional to $(\mathrm{V} / \mathrm{AL})$, for a fixed value of $\mathrm{L}$, this result also means that lower energy protons have a larger mean free path. On the other hand, comparison of Figures 3 e and 5 shows that electrons propagate more diffusively than protons. Figure 5 also shows that the injection profile broadens as the velocity of the particles decreases.

Although the model gave a good account of the time profiles for 30 out of a total of 31 western hemisphere events, a single exception occurred on March 12, 1969. The identification of the flare was based on strong associations with $\mathrm{H} \alpha, \mathrm{X}$-ray emission, type II, III, and IV radio bursts, and intense microwave bursts. But the proton profiles between 20 and $80 \mathrm{MeV}$, shown in Figure 6 , deviate significantly from predicted profiles given by the dashed curves. The best estimates of (V/AL) indicate that protons at higher energies are more coherent than those at lower energies. This feature, which disagrees with the behavior of other events, has no satisfactory 
explanation at the present time.

The analysis shows that the theory of focused diffusion combined with a Gaussian injection profile accounts satisfactorily for the time histories of both proton and electron events. The ratio (V/AL) is, on the one hand, smaller for electrons than for protons, and on the other hand, largex for lower energy protons than for higher energy ones. This result has an important bearing on the propagation of low energy particles in intexplanetary space. The model is especially successful in describing the scatter-free microevents, characterized by a very sharp time profile with a rapid xise and decay (Lin et al. 1973; Lin 1974; McDonald and Van Hollebeke 1973), as shown in Figures 3 and 4. Classical diffusion theory is totally inadequate in explaining these events, but they are successfully explained by the theory of focused diffusion, indicating that the coherent effect dominates over scattering in such cases. 


\section{RESULLTS AND DISCUSSION}

Detailed information on (V/AC) and the Gaussian injection width for $30^{\circ}$ events from $\sim W 20^{\circ}$ to $W 100^{\circ}$ is given in Table I. Figure 7 shows, for 18 events, the frequency distribution of (V/AL) for $0.5-1.1 \mathrm{MeV}$ electrons, 30-50 MeV protons, and 9-19 MeV protons. Excluded from this distribution are events for which the analysis could not be performed simultaneously for all three of these particle categories. It is apparent that (V/AL) is generally smaller for electrons than it is for protons. Similarly, for 9-19 MeV protons, the distribution, which extends to relatively large values of (V/AL), is broader than it is for $30-50 \mathrm{MeV}$ protons.

Figure 8 shows six examples of the mean free-path $\lambda$, calculated from equation (4) for individual events, as a function of rigidity for electrons and protons. Here, data at $R \geq 100 \mathrm{MV}$ refer to protons, white those at $\mathrm{R}=1^{\circ}$ and $8 \mathrm{MV}$ refer to $0.5-1.1$ and $3-12 \mathrm{MeV}$ eleetrons, respectively. In two cases, the events on September 29, 1968 and November 24, 1969, proton data were extended to $326 \mathrm{MeV}$ (rigidities up- to $847 \mathrm{MV}$ ) using an essentially identical. detector on $0 G 0 \mathrm{~V}$ which was described by Jones et al. (1967). Clearly, the rigidity dependence of $\lambda$ for protons varies from event to event, but, between 100 and $800 \mathrm{MV}$, the mean free path either stays relatively constant ox increases with decreasing proton rigidity. This behavior is also exhibited in the average $\lambda$ over all events shown in Figure 9, which includes all events except the microevents. The microevents were excluded because their Iimited number and incomplete analysis would create a bias. The error bars in Figure 8 give the standard deviation of the average. Note that for protons between 4 and $80 \mathrm{MeV}(\mathrm{R}=100-400 \mathrm{MV}), \lambda$ increases slowly with decreasing rigidity. For protons in this range, a typical mean 
free path is $00.2 \mathrm{AJ}$. The $1 \mathrm{MV}$ electrons have a mean free path approximately 2 to 3 times smaller.

The inverse relationship between the mean free path and particle rigidity, which has also been reported by Wibberenz (1977) and ZwickI

(1976), is in strong-disagreement with quasilinear theory of pitch-angle scattering. It follows from equations (2) and (4) above that $\lambda \propto R^{2-q}$ (Jokipii 1971). Thus, for $q<2$, the quasilinear theory predicts, in disagreement with the observed dependence, that the mean free path decreases with decreasing rigidity. On the other hand, if the experimental result that $\lambda$ either stays constant or increases with decreasing proton rigidity is interpreted within the context of quasilinear theory to mean that $q \geq 2$, then purely coherent propagation (Earl 1974a) is predicted but not observed. Moreover, the diffusion coefficient calculated following Jokipii and Coleman (1968) from the measured interplanetary magnetic field spectrum is approximately one order of magnitude smaller than the observed value of $\sim(0.6-1.2) \times 10^{22} \mathrm{~cm}^{2} / \mathrm{sec}$ at $40 \mathrm{MeV}$.

The paradox is further compounded at low rigidities by the electron data, which do not conform to the monotonic inverse dependence found for protons, but which suggest instead that the scattering length is a complicated function of rigidity which goes through a maximum somewhere between 10 and $500 \mathrm{MV}$, and then dips to minimum somewhere above $1000 \mathrm{MV}$. This implication must be taken very seriously, for it follows from the nature of the Lorentz force that the scattering length, which characterizes the effect of a static magnetic field, must be a function of rigidity alone. In the absence of a definitive theory, a complicated dependence of pitch-angle scattering upon rigidity cannot be ruled out a priori. Nevertheless, because this complexity seems implausible, it is worth considering alternatives that retain a simple dependence. One possibility is that both of the parameters, $q$ and $A$, which specify $\phi$ in equation (2), are functions 
of rigidity. This hypothesis, that the anisotropy of scattering, as we11 as the amplitude, depends upon rigidity, means that the scattering length (V/A) for a given mean free path $\lambda$ increases as q decreases (see eq. 4). Figures 8 and 9 show that the mean free path of electrons is smallex than that of protons. If $\mathrm{q}$ is held fixed at $\mathrm{q}=1.5$, as it was in' the above analysis, this also means that $(\mathrm{V} / \mathrm{A})$ is smaller for electrons than protons. But if scattering at the low rigidities of the electrons is more isotropic than it is at the rigidities of the protons, which are two orders of magnitude larger, then two different mean free paths could correspond to the same scattering length. For example, if $q=1.0$ for electrons. $(i, e, \lambda=V / A)$ and if $q=1.5$ for protons (i.e. $\lambda=2.4 \mathrm{~V} / \mathrm{A}$ ), then the electron mean free path, for a given (V/A), is a factor of 2.4 smaller than that of protons. This number is close enough to the observed ratio to make tenable an interpretation of the maximum in the $\lambda$ vs. rigidity curve as the effect of a slow increase of $q$ superposed upon a slow decrease of (V/A). However, this interpretation is not very satisfying, for it offers no fundamental explanation of the postulated rigidity dependences.

To seek further alternatives that embody monotonic dependences, it is appropriate to express the results appearing in Figure 9 as the following power law in the two particle parameters intrinsic to electromagnetic forces, the velocity $V$ and the charge to mass ratio $(Q / m)$ :

$$
\frac{V}{A} \propto\left(\frac{Q}{m}\right)^{-0.1} v^{-0.3} \text {. }
$$

In this expression, the dependence on $(Q / m)$ is weak enough to suggest a scattering process that depends on velocity alone. If the relationship $R=(m V / Q)$ is invoked to convert to a power $I$ aw in $(Q / m)$ and rigidity $R$, 
the result is:

$$
\frac{V}{A} \propto R^{-0.3}(Q / m)^{-0.4}
$$

Because scattexing by static magnetic fields must depend on rigidity alone, the presence here of a factor in $(\mathrm{Q} / \mathrm{m})$ demonstrates again the conclusion reached earlier that scattering is not a simple function of rigidity. Although the presence of electrical forces in interplanetary space seems very unlikely, they constitute the only other possible influence on charged particle trajectories. To investigate this possibility, we express the power law in terms of the kinetic energy per unit charge (E/Q),

$$
\frac{V}{A} \propto(E / Q)^{-0.15}(Q / m)-0.25
$$

Here again, the dependence embodies a factor in $(Q / m)$ which is not consistent with the pure $(\mathrm{E} / \mathrm{Q})$ dependence expected if the scattering is produced by electric fields alone. These considerations lead back to the conclusion that a non-monotonic dependence of scattering length on rigidity is the most plausible explanation of our results.

Many investigators have analyzed propagation in terms of the distance travelled by particles from the time of flare acceleration. For example, Bryant et a1. (1965) found that, during the eyent on September 28, 1961, 2-300 MeV protons shared the same probability distribution in distance travelled, and concluded that propagation during this event was rigidity independent (i.e. $K^{\propto} \beta \mathrm{R}^{\circ}$ ). Reinhard and Wibberenz (1974), and Ma Sung et al. (1975) showed that, at the time of maximum intensity, distance travelled could be expressed as a linear function of velocity,

$$
\mathrm{V} \Delta \mathrm{T}_{\max }=\mathrm{C}_{\max }+\mathrm{B}_{\max } \mathrm{V}
$$

where $\Delta \mathrm{T}_{\max }=$ risetime to maximum intensity, where $C_{\max }$ was interpreted 
as the distance travelled in interplanetary space and where $B_{\max }$ was interpreted as the time spent in the corona. This interpretation is equivalent to the conclusion that the diffusion coefficient $\mathrm{K}$ is independent of rigidity. The present analysis indicates that the dependence of propagation upon rigidity. is weak, but not entirely negligible. Furthermore, it is not possible to separate the injection and intexplanetary effects, as was done in equation (7), because the coronal injection profile also displays a weak dependence on velocity.

In section IV, it was shown that the injection width $\sigma$ increases with decreasing particle velocity. For example, in Figure 3, for the event on April 6, 1971, $\sigma=0.2$ hour for electrons $(\beta=0.91)$. For protons, in Figure $5, \sigma=0.3$ hours at $50-80 \mathrm{MeV}(\beta=0.35)$, and increases to 1.0 hour at $6-9 \mathrm{MeV}(\beta=0.125)$. This is also evident in the frequency distribution of $\sigma$ shown in Figure 10, which is based on the same set of 18 events as Figure 7. The majority of events have an injection width $\sigma \dot{\delta}_{i}$ hour, but a few are characterized by a large $\sigma$ of several hours. For those events with $\sigma>1$ hour at $40 \mathrm{MeV}$, it was found that a Gaussian profile truncated at the 3o level gives a slower onset phase. than is observed. A Gaussian truncated at the $1.5 \sigma$ level appears to be more appropriate, but this change does not affect events with a narrow injection profile. Figure 11 shows five proton events with prolonged injections each in a different energy range. Injection widths up to 8 hours have been found for proton events. The finite duration of injection can only arise in the corona. However, the methods employed here do not distinguish between prolonged accelexation and continuous injection. Since no apparent correlation is found between the injection width and the duration of the type IV bursts, it is assumed hereafter that $\sigma$ characterizes the duration of particle release from the corona.

Figure 12 shows a plot of $\sigma$ versus particle velocity averagèd over.a11 
events with $\sigma$ sI hour at $40 \mathrm{MeV}$. Events with prolonged injection were excluded because their analysis was incomplete. The large magnitude of the error bars, which indicate the standard deviation of the average, arose from large variation from event to event, and not from the uncertainties in individual measurements of $\sigma$. Data at $\beta \geq 0.9$ refei to electrons, and the rest refer to protons. There appears to be a continuous variation of $\sigma$ as a function of the velocity, regardless of the rigidity.

Because knowledge of the coronal release process is very limited, we assume, for simplicity, that the injection width follows a power law in rigidity and velocity. Then the best fit to the data leads to

$$
\sigma \propto \mathrm{R}^{-0.07 \pm 0.06} \quad \beta^{-0.55 \pm 0.85},
$$

in which the rigidity dependence is negligible. The dashed curve in Figure 12 shows a fit for the data given by the relation: $\sigma \propto \beta^{-0.55}$.

Figure 13 shows two typical events, on September 29, 1968, and February 25, 1969, for which the relation given above appears to be appropriate. However, deviations do occur. Figure 14 shows two examples. During the event on Apri1 22, 1971, the injection width was constant over the range $0.1 \leq \beta \leq 0.9$. During the event on March 29, 1970, $\sigma$ exhibited a strong dependence on $\beta$, for $\sigma=0$ for 3-12 MeV electrons $(\beta=1.0)$, and $\sigma$ increases six-fold as proton velocity decreases by a factor of three. However, this event was not included in the average shown in Figure 12 , because $\sigma>1$ hour at $40 \mathrm{MeV}$. These examples illustrate variability in the coronal release. However, one feature of the coronal release remains unchanged throughout al1 events: the electrons have an injection width similar to that of protons above $40 \mathrm{MeV}$, even though their rigidity is $2 \frac{1}{2}$ orders of magnitude lower; on the other hand, low energy protons are generally associated with broader injection profiles than high energy protons. These tendencies 
suggest strongly that rigidity plays essentially no role in the release process.

The velocity dependent injection width implies that the mean escape rate $\eta$ also depends on velocity:

$$
\eta \quad=\frac{1}{2.35 \sigma} \propto \beta^{0.55} \text {, }
$$

where we approximated the duration of injection by the full width at half maximum of the Gaussian. This relation predicts that high energy proton fluxes in space should have a steeper longitudinal gradient than low energy ones. The absence of rigidity as a factor in coronal release severely limits the possible mechanisms for this process, as discussed by Newkirk and Wentzel(1978). In a separate paper by Ma Sung, Van Hollebeke, and McDonald (1978), this question will be examined by analyzing the chaxacteristics of eastern hemisphere solar events.

Because both coronal escape and interplanetary propagation depend on particle parameters, the energy spectrum measured at the time of maximum intensity at a given position in space does not necessarily reproduce the source spectrum. Interplanetary propagation tends to enhance fluxes at lower energies, because propagation of low energy particles is more coherent. On the other hand, the velocity dependent injection profile tends to enhance fluxes at higher energies. Neither effect is strongly dependent on particle parameters, and the two effects work in opposite directions. On the basis of a careful study of many events, we conclude that the combined process alters the source spectrum by no moxe than 5\% at 1 AU. This analysis provides evidence that the average proton energy spectrum that was measured by Van Hollebeke et al. (1975), for events in $w 20^{\circ}$ to $W 80^{\circ}$, is identical to the source spectrum to within $5 \%$. 
Because the predictions of the model accurately describe the entire evolution of particle events from the western hemisphere, the time of particle release from the sun can be determined from the model, as listed in column 7 of Table I. By comparing this release time with the onset of the 2-20 $\AA$ soft $X$-ray bursts (listed in column 3 of Table I), we found that, with only one exception; no. delay occurred between the predicted particle release time, and the soft $X$-ray onset, provided that the onset time of $X$-ray. emission closely coincides with the onset time of type II and type IV radio bursts or intense microwave bursts. In the single exception, on June 29, 1971, the predicted release of protons occurred 3 hours after the occurrence of X-ray emission and type II and IV bursts. Four other events show significant delay in particle release compared to $X$-ray onset. of these, three events, on January 28, 1970, January 29, 1970, and Apri1 22, 1971, were associated with neither spectral radio bursts nor microwave bursts. Because flare assocation for these events was weak, the delays are probably not meaningful. Thẹ fourth event, on March 23, 1970, shows a delay of 2 hours if the predicted particle release time is compared with the X-ray onset, but shows no delay if it is. compared with the onset of type IV bursts, which occurred 2 hours after the onset of soft $X$-ray emission. Thus, in almost all cases, particle release begins at flare acceleration indicated by the concurrent outbursts of Ha emission, soft X-ray emission, type II and IV bursts, and microwave bursts. 


\section{SUMMARY AND CONCLUSION}

In this paper, a propagation model, which combines a Gaussian profile for particle release from the sun, with interplanetary particle densities predicted by the theory of focused diffusion, meets with remarkable success in describing the time-intensity profiles of 30 flare associated proton and electron events from the western hemisphere of the sun. This model makes possible a clear separation_of coronal and interplanetary effects. By comparing the predictions of the model with observed events over a wide range of propagation conditions and particle rigidities, we obtained the results stmmarized in the next two paragraphs.

The scattering mean free path $\lambda$ for flare associated particles is large. Its magnitude is on the order of 0.1 to $0.3 \mathrm{AU}$ for $4-80 \mathrm{MeV}$ protons, and a factor of 2 to 3 smaller for $0.5-1.1 \mathrm{MeV}$ and $3-12 \mathrm{MeV}$ electrons. Because the rigidities here differ by more than two orders of magnitude, this result indicates that $\lambda$ is only weakly dependent on rigidity. The mean free path is at least one order of magnitude larger than is predicted by the quasilinear theary of pitch-angle scattering. Furthermore, $\lambda$ exhibits a complex dependence on rigidity that is in strong disagreement with quasilinear theory,

The release of particles into interplanetary space generally occurs over a finite time interval. If the injection profile is approximated by a truncated Gaussian, then the r.m.s, width $\sigma$ is less than 1 hour except for a few events in which it can be as large as several hours. The injection width decreases with velocity, but it does not depend on rigidity. In almost all events, the release of particles occurs at the time of flare acceleration indicated by concurrent events in $\mathrm{H} \alpha$ emission, X-ray emission, 
type II, III, and IV radio bursts, and intense microwave bursts.

\section{Acknowledgement}

L.S. Ma Sung acknowledges the support of NASA/Goddard Space Flight Centex Grant NGR 21-002-3I6 while this research was carried out, and is grateful to Dr. F.B. McDonald and Dr. D.G. Wentzel for many helpful discussions and to Dr. M.A.I. Van Hollebeke for her generous assistance in the initial treatment of the data during the course of this analysis. The work of J.A. Earl is supported by NASA Grant NGR 21-002-066. 
Table I. SOLAR PARTICLE EVENTS - INTERPLANETARY PROPAGATION MODEL FITTING

\begin{tabular}{|c|c|c|c|c|c|c|c|}
\hline DATE & LONGITUDE & $\begin{array}{l}\text { ACCEL. } \\
\text { TIME } \\
\text { (UT) }\end{array}$ & $\begin{array}{c}V \\
(\mathrm{AU} / \mathrm{HR})\end{array}$ & $\frac{V}{A L}$ & $\sigma(H R)$ & $\begin{array}{l}\mathrm{T}_{\text {inj }} \\
(\mathrm{UT})\end{array}$ & $\begin{array}{l}\text { PARTICLE } \\
\text { SPECIES }\end{array}$ \\
\hline $5 / 28 . / 67$ & W32 & 5.35 & $\begin{array}{l}6.55 \\
2.52 \\
1.98 \\
1.58 \\
1.15 \\
0.79\end{array}$ & $\begin{array}{l}- \\
0.10 \\
0.12 \\
0.12 \\
0.14 \\
0.16\end{array}$ & $\begin{array}{l}0 \\
0.15 \\
0 \\
0.4 \\
0.8\end{array}$ & $\begin{array}{l}5.9 \pm 0.5 \\
5.4 \pm 0.5 \\
5.8 \pm 0.5 \\
5.2 \pm 0.5 \\
4.55 \pm 0.5\end{array}$ & $\begin{array}{l}\mathrm{e} \\
\mathrm{p} \\
\mathrm{p} \\
\mathrm{p} \\
\mathrm{p} \\
\mathrm{p}\end{array}$ \\
\hline $8 / 3 / 67$ & W85 & 9.13 & $\begin{array}{l}6.55 \\
1.98 \\
1.58\end{array}$ & $\begin{array}{l}0.14 \\
0.46 \\
0.50\end{array}$ & $\begin{array}{l}0.3 \\
0.4 \\
0.4\end{array}$ & $\begin{array}{l}9.2 \pm 0.25 \\
9.5 \pm 0.5 \\
9.3 \pm 0.5\end{array}$ & $\begin{array}{l}e \\
p \\
p\end{array}$ \\
\hline $12 / 3 / 67$ & W105 & 8.78 & $\begin{array}{l}6.55 \\
2.52 \\
1.98 \\
1.58 \\
1.15 \\
0.79\end{array}$ & $\begin{array}{l}0.03 \\
0.08 \\
0.10 \\
0.10 \\
0.12 \\
0.16\end{array}$ & $\begin{array}{l}0.2 \\
0.4 \\
0.4 \\
0.4 \\
0.6 \\
0.6\end{array}$ & $\begin{array}{l}8.2 \pm 0.25 \\
8.9 \pm 0.5 \\
9.4 \pm 0.5 \\
8.9 \pm 0.5 \\
8.3 \pm 0.5 \\
8.55 \pm 0.5\end{array}$ & $\begin{array}{l}\mathrm{e} \\
\mathrm{p} \\
\mathrm{p} \\
\mathrm{p} \\
\mathrm{p} \\
\mathrm{p}\end{array}$ \\
\hline $12 / 29 / 67$ & $\begin{array}{r}\text { W77 } \\
.\end{array}$ & 0.63 & $\begin{array}{l}6.55 \\
1.98 \\
1.58 \\
1.15 \\
0.79\end{array}$ & $\begin{array}{c}0.48 \\
0.85 \\
- \\
20.75 \\
0.75\end{array}$ & $\begin{array}{l}0 \\
0 \\
0 \\
0\end{array}$ & $\begin{array}{l}0.95 \pm 0.1 \\
0.95 \pm 0.25 \\
1.4 \pm 0.25 \\
2.1 \pm 0.25\end{array}$ & $\begin{array}{l}e \\
p \\
p \\
p \\
p\end{array}$ \\
\hline $2 / 17 / 67$ & \$47 & 2.72 & $\begin{array}{l}6.55 \\
2.52 . \\
1.98 \\
1.58 \\
1.15 \\
0.79\end{array}$ & $\begin{array}{l}- \\
0.18 \\
0.18 \\
0.18 \\
0.20 \\
0.24- \\
0.26\end{array}$ & $\begin{array}{l}0.4 \\
0.4 \\
0.4 \\
0.6 \\
0.6\end{array}$ & $\begin{array}{l}2.3 \pm 0.5 \\
2.3 \pm 0.5 \\
2.25 \pm 0.5 \\
2.1 \pm 0.5 \\
2.3 \pm 0.5\end{array}$ & $\begin{array}{l}e \\
p \\
p \\
p \\
p \\
p\end{array}$ \\
\hline $8 / 14 / 68$ & W80 & 13.28 & $\begin{array}{l}6.55 \\
2.52 \\
1.98 \\
1.58 \\
1.15 \\
0.79\end{array}$ & $\begin{array}{c}0.09 \\
0.20 \\
0.20 \\
0.20 \\
0.30 \\
-\end{array}$ & $\begin{array}{l}0.6 \\
0.8- \\
1.0 \\
0.8- \\
1.0 \\
0.8 \\
1.0\end{array}$ & $\begin{array}{l}12.7 \pm 0.25 \\
13.0 \pm 0.75 \\
12.8 \pm 0.5 \\
12.7 \pm 0.5 \\
12.4 \pm 0.5\end{array}$ & $\begin{array}{l}\mathrm{e} \\
\mathrm{p} \\
\mathrm{p} \\
\mathrm{p} \\
\mathrm{p} \\
\mathrm{p}\end{array}$ \\
\hline
\end{tabular}

The exror in (V/AL) is \pm 0.01 , and the exror in $\sigma$ is $\pm 0.1 \mathrm{hr}$.

The acceleration time is assumed to be at the onset of $2-20 \AA \mathrm{X}$-ray burst. 
TABLE I. (Con't $t$ )

\begin{tabular}{|c|c|c|c|c|c|c|c|}
\hline DATE & LONGITUDE & $\begin{array}{l}\text { ACCEL. } \\
\text { TTME } \\
\text { (UT) }\end{array}$ & $\begin{array}{c}V \\
\text { (AU/HR) }\end{array}$ & $\frac{V}{A \bar{L}}$ & $\sigma(\mathrm{HR})$ & $\begin{array}{l}\mathrm{T}_{\text {inj }} \\
\text { (UT) }\end{array}$ & $\begin{array}{l}\text { PARTICLE } \\
\text { SPECIES }\end{array}$ \\
\hline \multirow[t]{10}{*}{$9 / 29 / 68$} & T52 & 16.15 & 6.55 & 0.05 & 0.4 & $15.7 \pm 0.25$ & e \\
\hline & & & 4.68 & $\begin{array}{l}0.10- \\
0.12\end{array}$ & 0.4 & $15.9 \pm 1.0$ & $\mathrm{p}$ \\
\hline & & & 4.32 & $\begin{array}{l}0.10- \\
0.12\end{array}$ & 0.4 & $15.9 \pm 1.0$ & $\mathrm{p}$ \\
\hline & & & 3.74 & $\begin{array}{l}0.10- \\
0.12\end{array}$ & 0.6 & $15.9 \pm 1.0$ & $\mathrm{p}$ \\
\hline & & & 3.17 & 0.12 & 0.6 & $15.9 \pm 1.0$ & $\mathrm{p}$ \\
\hline & & & 2.52 & $\begin{array}{l}0.12- \\
0.14\end{array}$ & 0.6 & $15.9 \pm 0.5$ & $\mathrm{p}$ \\
\hline & & & 1.98 & $\begin{array}{l}0.12- \\
0.14\end{array}$ & 0.8 & $15.9 \pm 0.5$ & $\mathrm{p}$ \\
\hline & & & 1.58 & $\begin{array}{l}0.12- \\
0.14\end{array}$ & 0.8 & $.15 .8 \pm 0.5$ & $\mathrm{p}$ \\
\hline & & . & 1.15 & - & & & $\mathrm{p}$ \\
\hline & & & 0.79 & - & & & $\mathrm{p}$ \\
\hline \multirow[t]{7}{*}{$10 / 4 / 68$} & W37 & (03) 23.62 & 7.20 & 0.06 & 0.6 & $24.0 \pm 0.5$ & e \\
\hline & & & 6.55 & 0.06 & 0.6 & $23.6 \pm 0.25$ & e \\
\hline & & & 2.52 & 0.12 & 0.6 & $23.9 \pm 0.5$ & $\mathrm{p}$ \\
\hline & & & 1.98 & 0.12 & 0.6 & $23.9 \pm 0.5$ & $\mathrm{p}$ \\
\hline & & & 1.58 & 0.12 & 0.8 & $23.8 \pm 0.5$ & $\mathrm{p}$ \\
\hline & & & 1.15 & 0.12 & 1.0 & $23.3 \pm 0.5$ & $\mathrm{p}$ \\
\hline & & & 0.79 & - & & & $\mathrm{p}$ \\
\hline $10 / 31 / 68$ & W37 & (30) 23.52 & 1.58 & 0.20 & $\sim 6.0$ & $1.0 \pm 1.0$ & $\mathrm{p}$ \\
\hline \multirow[t]{5}{*}{$2 / 24 / 69$} & W32 & 22.9 & 6.55 & 0.08 & 0.3 & $22.5 \pm 0.4$ & e \\
\hline & & & 1.98 & 0.20 & 0.4 & $22.3 \pm 0.5$ & $\mathrm{p}$ \\
\hline & & & 1.58 & 0.20 & 0.6 & $22.7 \pm 0.5$ & $P$ \\
\hline & & & 1.15 & $\begin{array}{l}0.20- \\
0.22\end{array}$ & 0.6 & $22.1 \pm 0.5$ & $\mathrm{p}$ \\
\hline & & & 0.79 & 0.24 & 0.6 & $22.8 \pm 0.5$ & $\mathrm{p}$ \\
\hline \multirow[t]{7}{*}{$2 / 25 / 69$} & W37 & 8.80 & 7.20 & 0.07 & 0.3 & $8.7 \pm 0.5$ & e \\
\hline & & & 6.55 & 0.07 & 0.3 & $8.7 \pm 0.4$ & e \\
\hline & & & 2.52 & $\begin{array}{l}0.16- \\
0.18\end{array}$ & 0.4 & $8.8 \pm 0.5$ & $\mathrm{p}$ \\
\hline & & & 1.98 & $\begin{array}{l}0.16- \\
0.18\end{array}$ & 0.6 & $8.5 \pm 0.5$ & $p$ \\
\hline & & & 1.58 & $\begin{array}{l}0.16- \\
0.18\end{array}$ & 0.8 & $8.25 \pm 0.5$ & $\mathrm{p}$ \\
\hline & & & 1.15 & $\begin{array}{l}0.18 \\
0.20\end{array}$ & 0.8 & $8.1 \pm 0.5$ & $\mathrm{p}$ \\
\hline & & & 0.79 & - & & . & $\mathrm{p}$ \\
\hline
\end{tabular}


TABLE I. (Con't)

\begin{tabular}{|c|c|c|c|c|c|c|c|}
\hline DATE & LONGITUDE & $\begin{array}{l}\text { ACCEL. } \\
\text { TIME } \\
\text { (UT) }\end{array}$ & $\begin{array}{c}\mathrm{V} \\
(\mathrm{AU} / \mathrm{HR})\end{array}$ & $\frac{\mathrm{V}}{\mathrm{AL}}$ & $\sigma(\mathrm{HR})$ & $\begin{array}{l}\mathrm{T}_{\text {inj }} \\
\text { (UT) }\end{array}$ & $\begin{array}{l}\text { PARTICLE } \\
\text { SPECIES }\end{array}$ \\
\hline $2 / 27 / 69$ & W65 & 13.82 & $\begin{array}{l}6.55 \\
2.52 \\
1.98 \\
1.58 \\
1.15 \\
0.79\end{array}$ & $\begin{array}{l}0.02 \\
0.12 \\
0.16 \\
0.20 \\
0.26 \\
-\end{array}$ & $\begin{array}{l}0.8 \\
2.0 \\
3.0 \\
3.0 \\
4.0\end{array}$ & $\begin{array}{l}13.5 \pm 0.4 \\
13.9 \pm 0.5 \\
13.8 \pm 0.5 \\
14.5 \pm 0.5 \\
14.5 \pm 0.5\end{array}$ & $\begin{array}{l}\mathrm{e} \\
\mathrm{p} \\
\mathrm{p} \\
\mathrm{p} \\
\mathrm{p} \\
\mathrm{p}\end{array}$ \\
\hline $3 / 27 / 69$ & W68 & 13.18 & $\begin{array}{l}6.55 \\
2.52 \\
1.98 \\
1.58 \\
1.15 \\
0.79\end{array}$ & $\begin{array}{l}0.02 \\
0.06 \\
0.06 \\
0.06 \\
0.06 \\
-\end{array}$ & $\begin{array}{l}0.2 \\
0.8 \\
0.8 \\
0.8 \\
0.6\end{array}$ & $\begin{array}{l}13.1 \pm 0.25 \\
14.4 \pm 0.5 \\
13.9 \pm 0.5 \\
13.9 \pm 0.5 \\
13.9 \pm 0.5\end{array}$ & $\begin{array}{l}e \\
p \\
p \\
p \\
p \\
p\end{array}$ \\
\hline $9 / 25 / 69$ & W14 & 6.50 & $\begin{array}{l}6.55 \\
2.52 \\
1.98 \\
1.58 \\
1.15 \\
0.90\end{array}$ & $\begin{array}{l}0.07 \\
0.20 \\
0.20 \\
0.20 \\
0.22 \\
0.22\end{array}$ & $\begin{array}{l}0.2 \\
0.4 \\
0.6 \\
0.6 \\
0.6 \\
0.6\end{array}$ & $\begin{array}{l}7.0 \pm 0.25 \\
6.8 \pm 0.5 \\
6.8 \pm 0.5 \\
6.7 \pm 0.5 \\
6.1 \pm 0.5 \\
6.5 \pm 0.5\end{array}$ & $\begin{array}{l}\mathrm{e} \\
\mathrm{p} \\
\mathrm{p} \\
\mathrm{p} \\
\mathrm{p} \\
\mathrm{p}\end{array}$ \\
\hline $11 / 24 / 69$ & W31 & 9.17 & $\begin{array}{l}6.55 \\
3.74 \\
3.17 \\
2.52 \\
1.98 \\
\\
1.58 \\
1.15 \\
0.90\end{array}$ & $\begin{array}{l}0.02 \\
0.06 \\
0.06 \\
0.06 \\
0.06- \\
0.08 \\
0.08 \\
0.10- \\
0.12 \\
0.12- \\
0.14\end{array}$ & $\begin{array}{r}0.2 \\
\leq 0.2 \\
\leq 0.2 \\
0.2 \\
0.2 \\
\\
0.2 \\
0.6 \\
\\
0.6\end{array}$ & $\begin{array}{l}8.0 \pm 0.25 \\
9.0 \pm 1.0 \\
9.0 \pm 1.0 \\
8.9 \pm 0.5 \\
8.9 \pm 0.5 \\
8.9 \pm 0.5 \\
9.8 \pm 0.5 \\
9.7 \pm 0.5\end{array}$ & $\begin{array}{l}\mathrm{e} \\
\mathrm{p} \\
\mathrm{p} \\
\mathrm{p} \\
\mathrm{p} \\
\mathrm{p} \\
\mathrm{p} \\
\mathrm{p}\end{array}$ \\
\hline $12 / 30 / 69$ & W85 & 18.92 & $\begin{array}{l}2.52 \\
1.98 \\
1.58\end{array}$ & $\begin{array}{l}0.12 \\
0.12 \\
0.12\end{array}$ & $\begin{array}{l}2.0 \\
3.0 \\
2.0\end{array}$ & $\begin{array}{l}19.4 \pm 0.5 \\
18.9 \pm 0.5 \\
19.5 \pm 0.5\end{array}$ & $\begin{array}{l}p \\
p \\
p\end{array}$ \\
\hline $1 / 28 / 70$ & W26 & 10.22 & $\begin{array}{l}6.55 \\
2.52 \\
1.98 \\
1.58 \\
1.15 \\
0.90\end{array}$ & $\begin{array}{c}0.04 \\
0.18 \\
- \\
0.22 \\
- \\
-\end{array}$ & $\begin{array}{l}0.2 \\
1.0 \\
\\
2.0\end{array}$ & $\begin{array}{c}12.5 \pm 0.25 \\
13.8 \pm 0.5 \\
14.2 \pm 0.5 \\
-\end{array}$ & $\begin{array}{l}e \\
p \\
p \\
p \\
p \\
p\end{array}$ \\
\hline
\end{tabular}


TABLE I. (Cont'd)

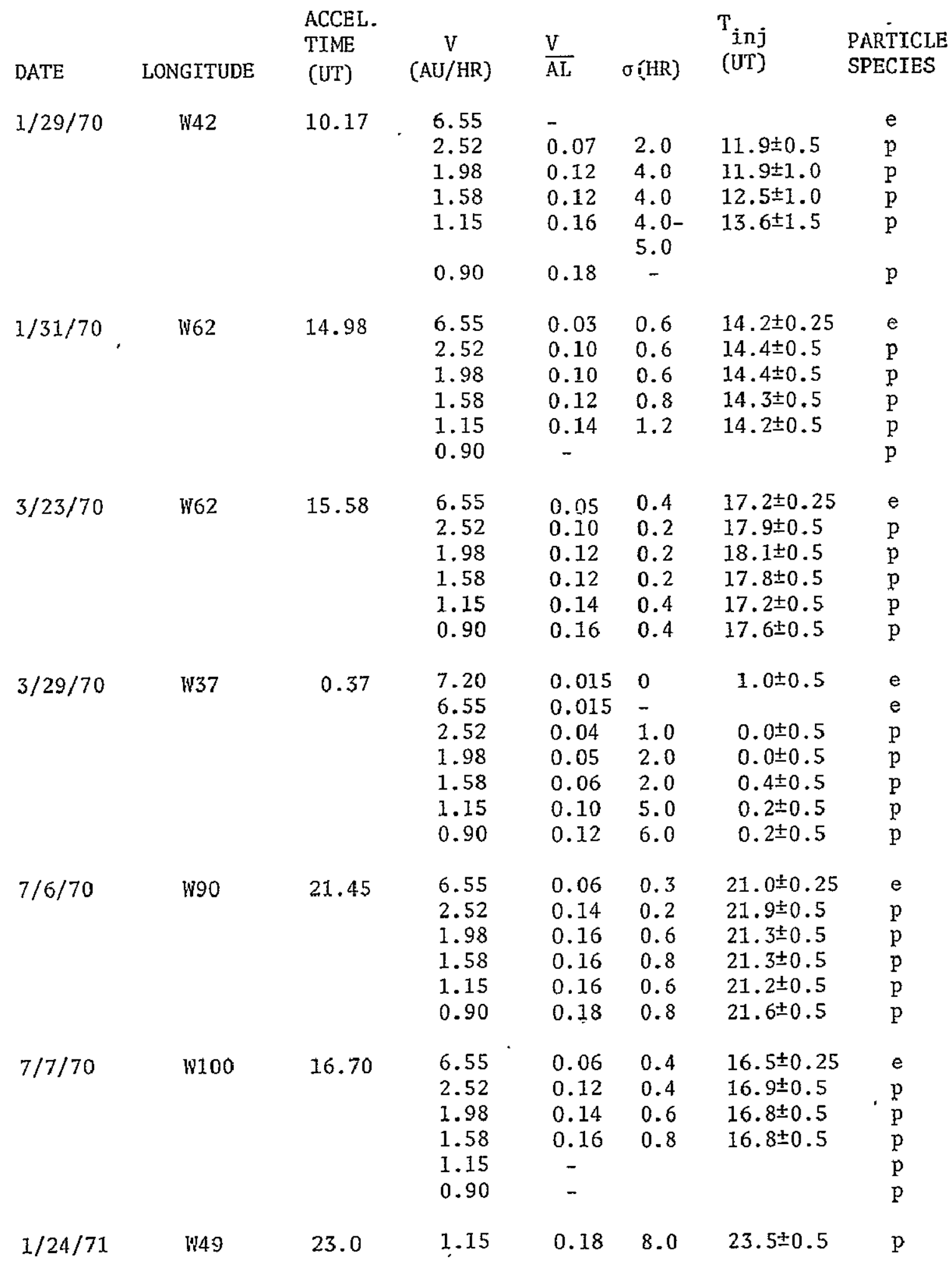


TABLE I. (Cont'd)

\begin{tabular}{|c|c|c|c|c|c|c|c|}
\hline DATE & LONGITUDE & $\begin{array}{l}\text { ACCEL. } \\
\text { TIME } \\
\text { (UT) }\end{array}$ & $\begin{array}{c}\mathrm{V} \\
(\mathrm{AU} / \mathrm{HR})\end{array}$ & $\frac{\mathrm{V}}{\mathrm{AL}}$ & $\sigma(H R)$ & $\begin{array}{c}T_{\text {inj }} \\
(U T)\end{array}$ & $\begin{array}{l}\text { PARTICLE } \\
\text { SPECIES }\end{array}$ \\
\hline $4 / 6 / 71$ & W80 & 9.42 & $\begin{array}{l}6.55 \\
2.52 \\
1.98 \\
1.58 \\
1.15 \\
0.90\end{array}$ & $\begin{array}{l}0.04 \\
0.12 \\
0.14 \\
0.16- \\
0.18 \\
0.18 \\
0.20\end{array}$ & $\begin{array}{l}0.2 \\
0.3 \\
0.4 \\
1.4 \\
\\
1.0 \\
1.0\end{array}$ & $\begin{array}{r}9.4 \pm 0.4 \\
9.9 \pm 0.5 \\
9.8 \pm 0.5 \\
9.2 \pm 0.5 \\
9.9 \pm 0.5 \\
10.0 \pm 0.5\end{array}$ & $\begin{array}{l}e \\
p \\
p \\
p \\
p \\
p\end{array}$ \\
\hline $4 / 20 / 71$ & $\$ 50$ & 19.32 & $\begin{array}{l}6.55 \\
2.52 \\
1.98 \\
1.58 \\
1.15 \\
0.9\end{array}$ & $\begin{array}{c}0.06 \\
- \\
0.20 \\
- \\
0.22 \\
-\end{array}$ & $\begin{array}{l}0.3 \\
0.6 \\
0.6\end{array}$ & $\begin{array}{l}19.0 \pm 0.25 \\
19.3 \pm 0.5 \\
20.1 \pm 0.5\end{array}$ & $\begin{array}{l}e \\
p \\
p \\
p \\
p \\
p\end{array}$ \\
\hline $4 / 22 / 71$ & W61 & 9.58 & $\begin{array}{l}6.55 \\
2.52 \\
1.98 \\
1.58 \\
1.15 \\
0.90\end{array}$ & $\begin{array}{l}0.06 \\
0.16 \\
0.16 \\
0.16 \\
0.20 \\
0.22\end{array}$ & $\begin{array}{l}0.6 \\
0.6 \\
0.6 \\
0.6 \\
0.6 \\
0.6\end{array}$ & $\begin{array}{l}10.7 \pm 0.25 \\
10.9 \pm 0.5 \\
10.8 \pm 0.5 \\
10.8 \pm 0.5 \\
10.6 \pm 0.5 \\
11.0 \pm 0.5\end{array}$ & $\begin{array}{l}\mathrm{e} \\
\mathrm{p} \\
\mathrm{p} \\
\mathrm{p} \\
\mathrm{p} \\
\mathrm{p}\end{array}$ \\
\hline $6 / 29 / 71$ & W22 & 22.45 & $\begin{array}{l}6.55 \\
2.52 \\
1.98 \\
1.58 \\
1.15 \\
0.90\end{array}$ & $\begin{array}{l}- \\
0.16 \\
0.16 \\
0.18 \\
0.20 \\
0.22\end{array}$ & $\begin{array}{l}0.4 \\
0.4 \\
0.6 \\
0.6 \\
1.0\end{array}$ & $\begin{array}{l}1.9 \pm 0.5 \\
1.8 \pm 0.5 \\
1.7 \pm 0.5 \\
1.1 \pm 0.5 \\
1.4 \pm 0.5\end{array}$ & $\begin{array}{l}\mathrm{e} \\
\mathrm{p} \\
\mathrm{p} \\
\mathrm{p} \\
\mathrm{p} \\
\mathrm{p}\end{array}$ \\
\hline $11 / 25 / 72$ & W44 & 8.20 & 6.55 & 0.11 & 0.2 & $9.0 \pm 0.25$ & e \\
\hline $11 / 28 / 72$ & W80 & 3.78 & $\begin{array}{l}6.55 \\
2.52 \\
1.98 \\
1.58 \\
1.15 \\
0.90\end{array}$ & $\begin{array}{l}0.09 \\
- \\
0.12 \\
0.12 \\
0.14 \\
-\end{array}$ & $\begin{array}{l}0.4 \\
0.6 \\
0.4 \\
0.6\end{array}$ & $\begin{array}{l}4.0 \pm 0.25 \\
3.4 \pm 0.5 \\
3.8 \pm 0.5 \\
3.7 \pm 0.5\end{array}$ & $\begin{array}{l}e \\
p \\
p \\
p \\
p \\
p\end{array}$ \\
\hline
\end{tabular}


Allum, F.R., Palmeira, R.A.R., Rao, U.R., McCracken, K.G., Haries, J.R., and- Palmer, I. 1971, Solar Phys. 17, 241.

Barouch, E., Gros, M., and Masse, P. 1971, Solar Phys. 19, 483.

Bieber, J.W. 1977, Ph.D. Thesis, University of Maryland.

Bryant, D.A., C1ine, T.L., Desai, U.D., and McDonald, F.B. 1962, J. Geophys.

Res. $67,4983$.

- 1965, Astrophys. J. 141, 478.

Burlaga, L.F. 1967, J. Geophys. Res. 22, 4449.

Countee, C.R. and Lanzerotti, L.J. 1976, J. Geophys. Res. 81, 441.

Datlowe, D. 1971, Solar Phys. 17, 436.

Ear1, J.A. 1974 a, Astrophys. J. 188, 379.

- 1974 b, Astrophys. J. 193, 231.

- 1976 a, Astrophys. J. 205, 900 .

- 1976 b, Astrophys. J. 206, 301 .

Earl, J.A., and Bieber, J.W. 1977, 15th Int. Cosmic Ray Conf. PIovdiv, Bulgaria, 5, 172 .

Englade, R.C. 1971, J. Geophys, Res. 76, 768.

Fan, C.Y., Pick, M., Pyle, R., Simpson, J.A., and Smith, D.R. 1968,

J. Geophys. Res. $\underline{73}, 1555$.

Feit, J. 1973, Solar Phys. 29, 211.

Hamilton, D.C. 1977, J. Geophys. Res. 82, 2157.

Jokipii, J.R. 1966, Astrophys. J. 146, 480.

. 1967, Astrophys. J. 149, 405.

- 1971, Rev. Geophys. and Space Phys. 9, 27.

Jokipii, J.R., and Coleman, P.J., Jr. 1968, J. Geophys. Res. 73, 5495.

Jokipii, J.R., and Parker, E.N. 1968, J. Geophys. Res. 73, 3367.

- 1969, Astrophys. J. 155, 777. 
Jones, S.L., Ludwig, G.H., Stilwel1, D.E., Trainór, J.H. , and 'way', S.H. 1967, IEEE Trans, Nuc. Sci. NS-14, 56.

Kinsey, J.H. 1970, Ph.D. Thesis, University of Maryland, NASA/GSFC X-611-69-396.

Kunstmann, J.E., and Alpers, W. 1977, Astrophys. J. 211, 587. Lanzerotti, L.J. 1973, J. Geophys. Res. 78, 3942 . Lanzerotti, L.J., Venkatesan, D., and Wibberenz, G. 1973; J. Geophys. Res. 78, 7986 .

Lin, R.P. 1974, Space Sci. Rev. 16, 189.

Lin, R.P., Evans, L.G., and Fainberg, J., 1973, Astrophys. Letters 14, 191. Lupton, J.E. and Stone, E.C. 1973, J. Geophys. Res. 78, 1007. Ma Sung, L.S. 1977, Ph.D. Thesis, University of Maryland, NASA/GSFC $\mathrm{X}-660-77-113$.

Ma Sung, L.S., Van Hollebeke, M.A., and McDonald, F.B. 1975, Pxoc. 14th Int. Cosmic Ray Conf., Munich, 5, 1767.

- 1978, in preparation.

McCracken, K.G., Rao, U.R., and Bukata, R.P. 1967, J. Geophys. Res. 72, 4293. McCracken, K.G., Rao, U.R., and Ness, N.F. 1968, J. Geophys. Res. 73, 4159. McDonald, F.B., and Van Hollebeke, M.A. 1973, Proc. Symp. on High Energy

Phenomena on the Sun, ed. R. Ramaty and R.G. Stone, NASA/GSFC $X-693-73-193$, p. 404.

McKibben, R.B. 1972, J. Geophys. Res. 77, 3957. Meyer, P., Parker, E.N., and Simpson, J.A. 1956, Phys. Rev. 104, 768. Newkixk, G., Jr., and Wentzel, D.G. 1978, to be published in J. Geophys. Res. Nie1sen, E., Pomerantz, M.A., and West, H.I., Jr. 1975, P1anet. Space Sci., 23. 1179 .

Ng, C.K., and Gleeson, L.J. 1976, Solar Phys., 46, 347.

Palmer, I.D., Palmeira, R.A.R., and Allum, F.R. 1975, Solar Phys., 40, 449. 
Parker, E.N. 1956, Phys. Rev. 103, $15-18$. - 1963, Interplanetary Dynamical Processes, John Wiley and Sons, New York.

Rao, U.R., McCracken, K.G., Allum, F.R., Palmeira, R.A.R., Bartley, W.C., and Palmer, I. 1971, Solar Phys. 19, 209.

Reinhard, R. 1975, Proc. 14th Int. Cosmis Ray Conf., Munich, ㅍ, 1687.

Reinhard, R., and Wibberenz, G. 1974, Solar Phys. 36, 473.

Roelof, E.C. 1966, Thesis, Univ. of California, Berkeley. - 1968, Canadian J. Phys, 46, S990.

Roelof, E.C. and Krimigis, S.M. 1973, J. Geophys. Res. 78, 5375.

Simnett, G.M. 1971, Proc. 12th Int. Cosmic Ray Conf., Hobart $\underline{7}, 2647$. Van Hollebeke, M.A.I., Ma Sung, L.S., and McDonald, F.B. 1975, Solar Phys. $\underline{41}, 189$.

Van Hollebeke, M.A.I., Wang, J.R., and McDonald, F.B. 1974, A Catalogue of Solar Cosmic Ray Events IMPS IV and V (May 1967-December 1972), NASA/GSFC $X-661-74-27$.

Webb, S., Balogh, A., Quenby, J.J. and Sear, J.F. 1973, Solar Phys. 29, 477. Webb, S. and Quenby, J.J. 1973, P1anet. Space Sci. 21, 23.

Wibberenz, G. 1977, Invited paper, L.D. deFeiter Memoraial symposium on the Study of Travelling Interplanetary Phenomena (STIP), Tel Aviv, Israe1, 7-10 June 1977.

Wibberenz, G., Lanzerotti, L.J., and Venkatesan, D. 1977, J. Geophys. Res. 81, 5807 .

Zwick1, R.D. 1976, Ph.D. Thesis, University of New Hampshire. 
FIGURE CAPTIONS

Figure 1 Comparison of density profiles for $40 \mathrm{MeV}$ protons $(\mathrm{V}=1.98 \mathrm{AU} / \mathrm{hr})$. The solid curves show the densities according to the convolution approximation given by equation (5), the dotted curves show those according to the accurate solution calculated by Bieber (1977), and the dashed curves show the contribution of the coherent Gaussian. The difference between the solid and dotted curves is not significant for both $(V / A L)=0.2$ and 0.7 . The contribution of the coherent Gaussian overshadows the inconspicuous tail of the diffusive wake when $(\mathrm{V} / \mathrm{AL})=0.7$, and it amounts to a significant fraction (36\%), of the total density at maximum even when $(V / A L)=0.2$ for which the diffusive wake dominates.

Figure 2 Comparison of the observed time intensity profiles of $0.5-1.1 \mathrm{MeV}$ electrons and 30-50 MeV protons with the calculated density proficles. The solid curves give profiles for the values of (V.AL) which best fit the observed particle density during the decay phases. The dashed. curves give profiles for two neighboring values of (V/AL). The value of $\sigma$ indicates the standard deviation of the Gaussian coronal injection. profile which best fits the onset-to-maximum phase of the event.

Figure 3 A sample of the 0.5-1.1 MeV electron events fitted by the calculated density profiles. The electron data were sampled every 3 minutes in (a), and were averaged over 15 minutes in the rest. The sample shows: (a) and (b), two scattex-free electrön events with Iarge (V/AL); (c). to (f), progressively more diffusive events with decreasing (V/AL); (g), an electron event at 3-12 MeV. The same event at 0.5-1.1 MeV is shown as the second event in $(\mathrm{d})$. 
Figure 4 A sample of the $40 \mathrm{MeV}$ proton events fitted by calculated densities. The proton data were averaged over 15 minutes in (a), and over 30 minutes in the rest. The sample.shows, (a) and (b), two scattex-free proton events with large (V/AL); (c) to (g), progressively more diffusive events with decreasing (V/AL)..

Figure 5 Comparison of the propagation characteristics of protons between 4 and $80 \mathrm{MeV}(\mathrm{R}=87-400 \mathrm{MV})$ for a typical western event. The solid curves give the best estimate of (V/AL) in each energy range, and the dashed curves show the variations about the best fit in steps of 0.02 in (V/AL). Note that (V/AL) increases with decreasing proton energy.

Figure 6 Proton time intensity profiles for the March 12, 1969 event. The dashed curves show the best possible fit according to Equation (6). The fit is poor for $>20 \mathrm{MeV}$ protons. There is no satisfactory explanation for this behavior.

Figure 7 Frequency distribution of (V/AL) for $180.5-1.1 \mathrm{MeV}$ electron, 30-50 $\mathrm{MeV}$ proton, and 9-19 MeV proton events. For electrons, (V/AL) is generally much smallex than it is for protons. The lower energy protons tend to have a broader distribution in (V/AL) than the higher energy ones do.

Figure 8 The scattering mean free path $\lambda$ as a function of rigidity $R$ for six electron and proton events. Data at $R \geq 100 \mathrm{MV}$ refer to protons above $4 \mathrm{MeV}$, and those at 1 and $8 \mathrm{MV}$ refer to $0.5-1.1$ and $3-12 \mathrm{MeV}$ electrons, respectively. Between 100 and $800 \mathrm{MV}$ the proton mean free path either stays constant or decreases with rigidity. The electron mean free path is generally a factor of 2 to 3 smaller than that of protons above $20 \mathrm{MeV}$.

Figure 9 The scattering mean free path $\lambda$, averaged over some 20 westem events, . as a function of particle rigidity $R$. The data point at $R=1 \mathrm{MV}$ refers 
to $0.5-1.1 \mathrm{MeV}$ electrons, while those above $100 \mathrm{MV}$ refer to protons above $4 \mathrm{MeV}$. The exror bars indicate the standard deviation of the average, rather than the uncertainties in the measurements. For protons between 4 and $80 \mathrm{MeV}, \lambda$ decreases with rigidity.

Figure 10 Frequency distribution of the Gaussian injection width $\sigma$ for $180.5-$ $1.1 \mathrm{MeV}$ electron, $30-50 \mathrm{MeV}$ proton, and 9-19 MeV proton events.

Figure 1̣ Five examples of proton events with prolonged injections each in a different energy range. Note that injection widths up to 8 hours have been found for proton events.

Figure 12 The Gaussian injection width $\sigma$ as a function of the particle velocity $\beta$ averaged over some 20 events. The data point at $\beta=0.9$ refers to 0.5-1.1 MeV electrons, and the rest refer to protons. The error bars show the standard deviation of the average. The dashed curve gives the best fit in the form, $\sigma \propto \beta^{-0.55}$. Long-injection events were not included in the averaging.

Figure 13 The Gaussian injection width $\sigma$ versus $\beta$ for two typical events. In each case, the relation $\sigma \propto \beta^{-0.55}$ appears to be appropriate. Data at $\beta=0.9$ and 1.0 refer to $0.5-1.1 \mathrm{MeV}$ and $3-12 \mathrm{MeV}$ electrons, respectively. The rest refer to protons ranging from 4 to $\sim 300 \mathrm{MeV}$ in energy.

Figure 14 The Gaussian injection width $\sigma$ versus $\beta$ for two extreme cases. $\sigma$ decreases steeply with particle velocity for the March 29, 1970 event, while it shows no variation as a function of $\beta$ for the ApriI 22, 1971 event. 


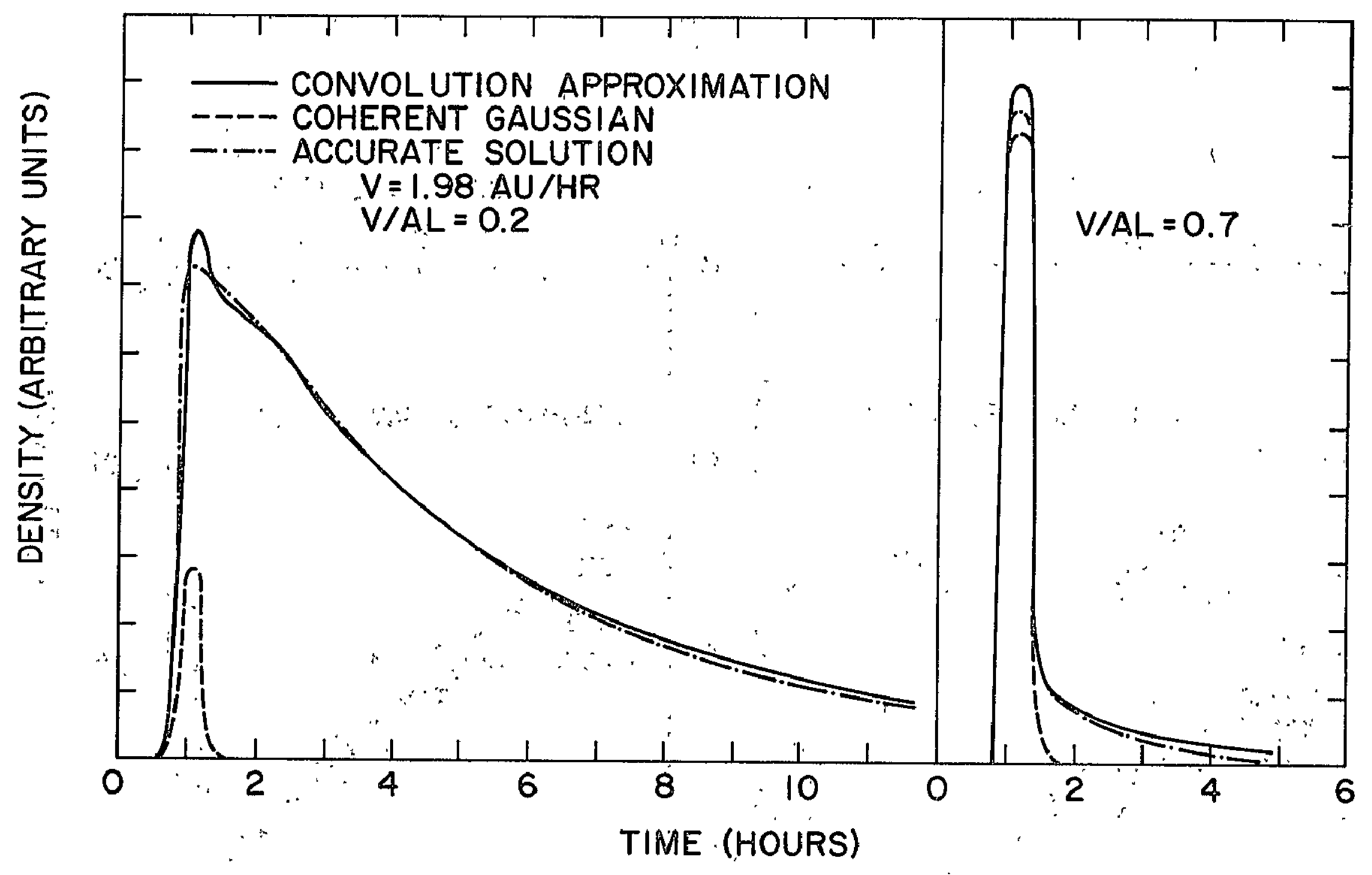

Figure I 


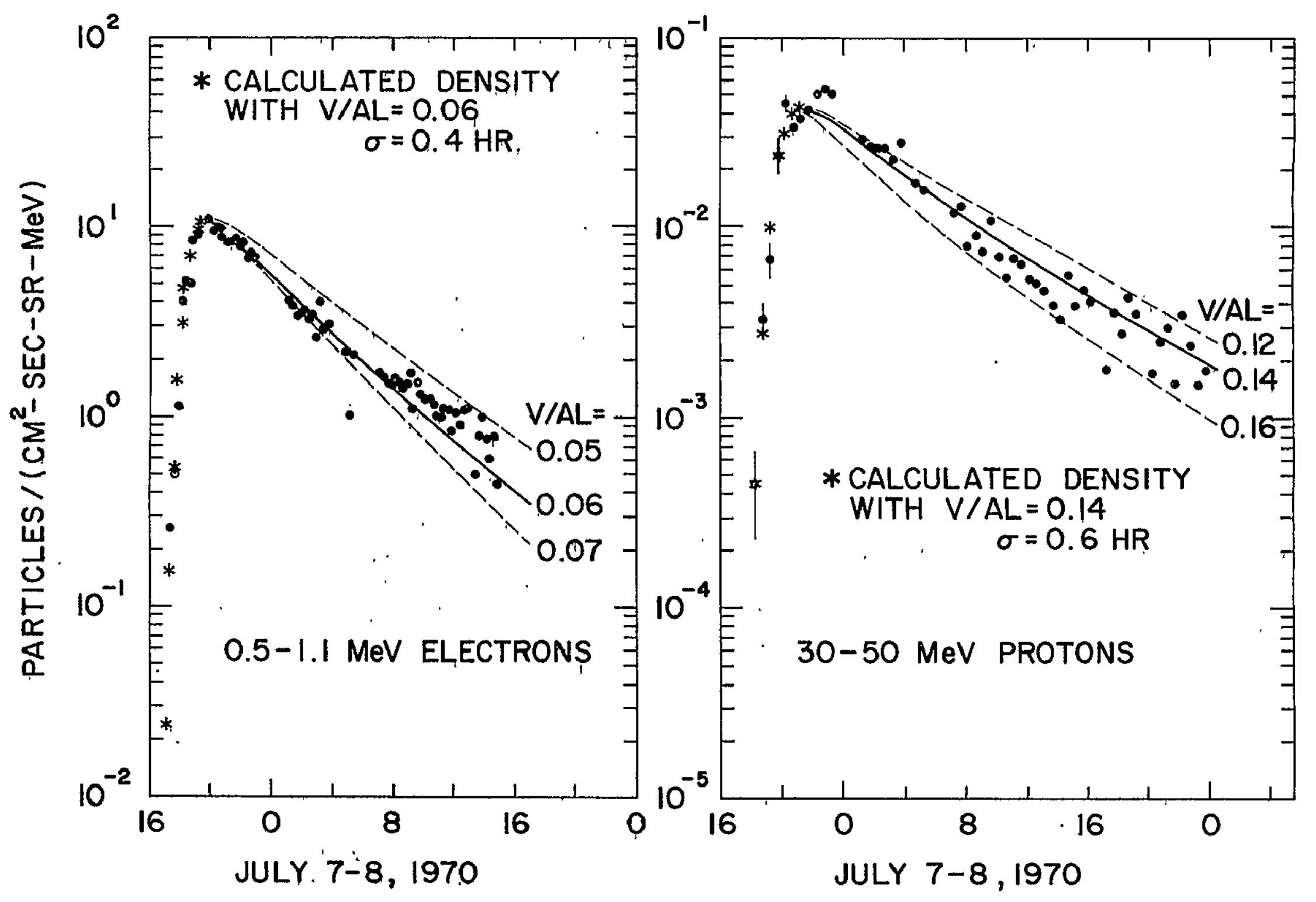

Figure 2 

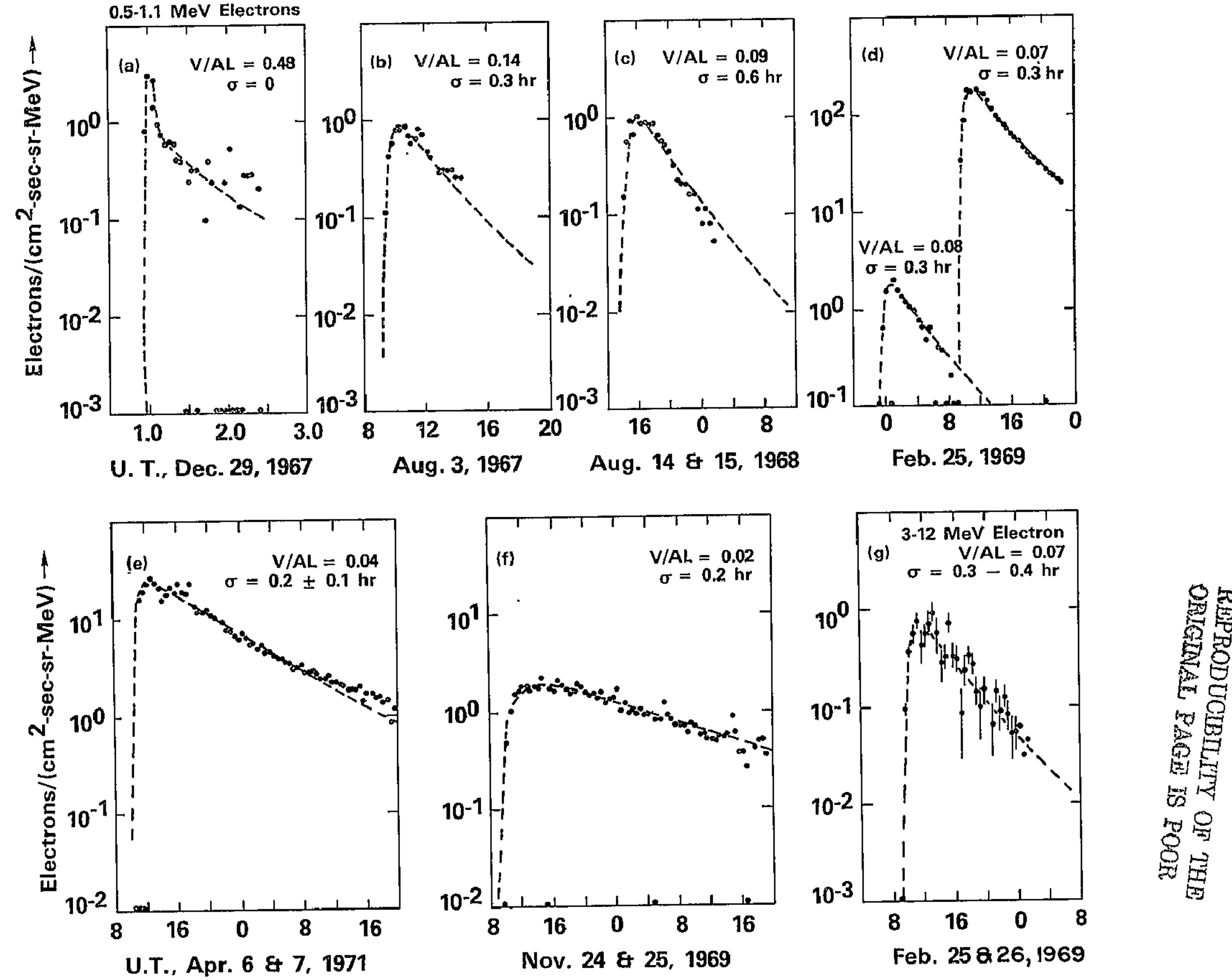

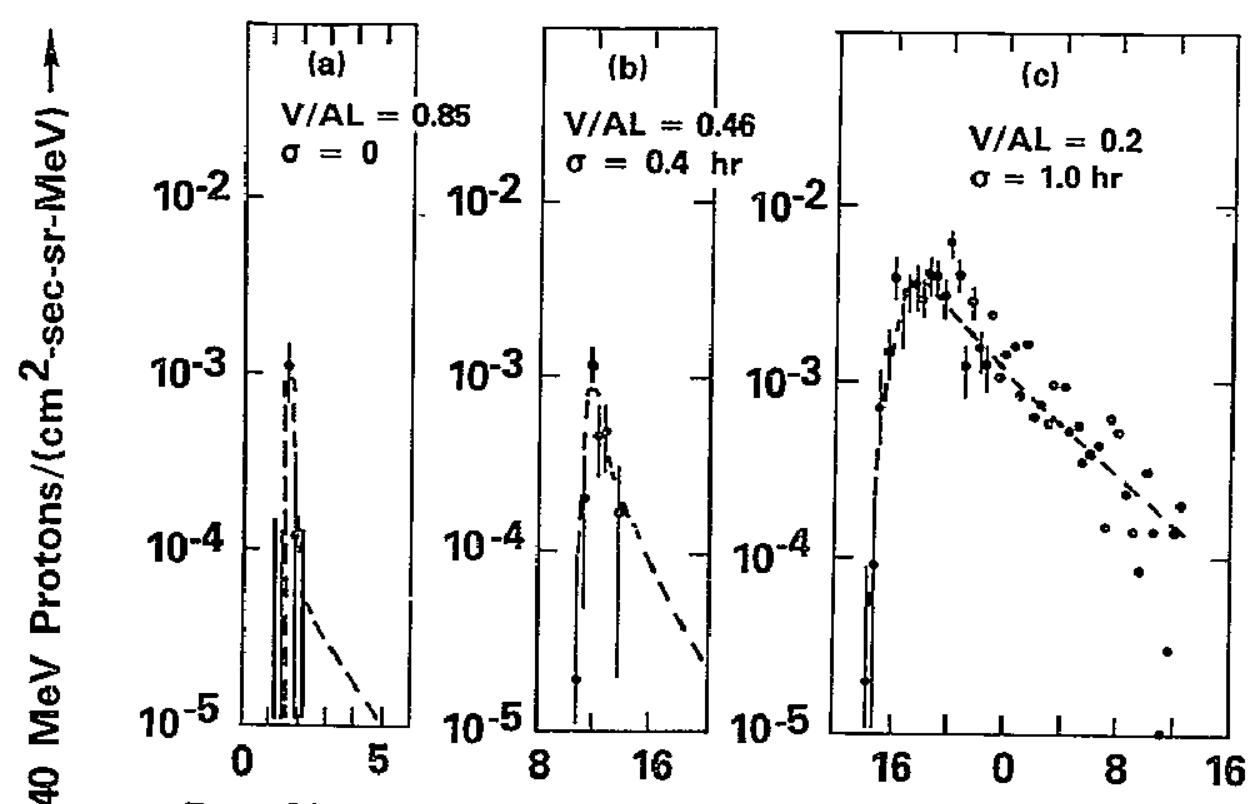

Dec. 29, 1967 Aug. 3, 1967

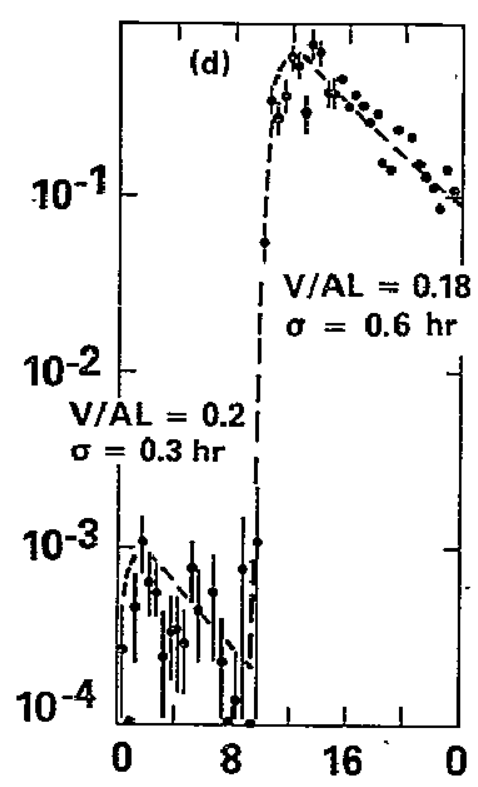

Aug. 14 \& 15, 1968

Feb. 25, 1969
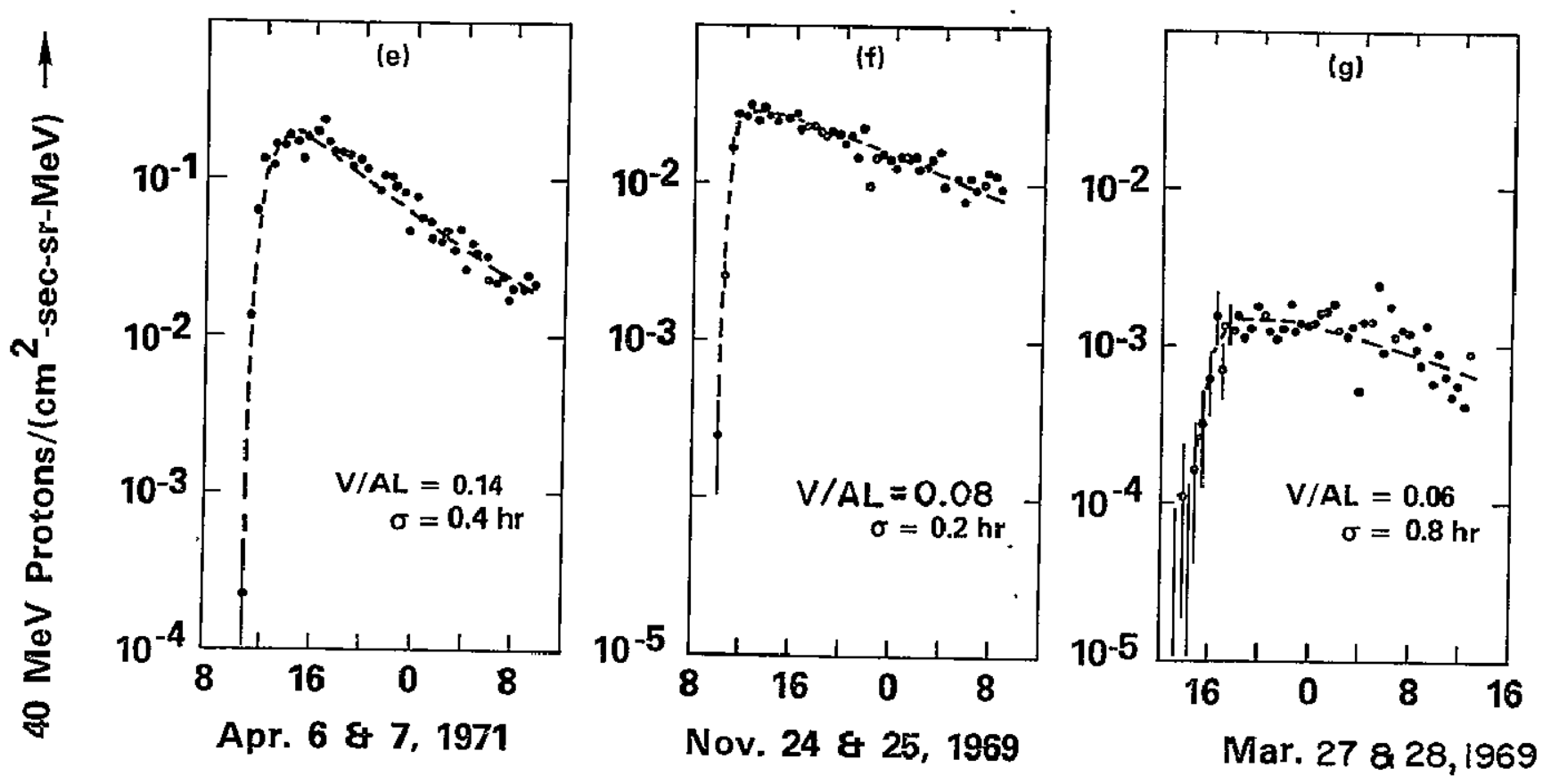

Figure 4 


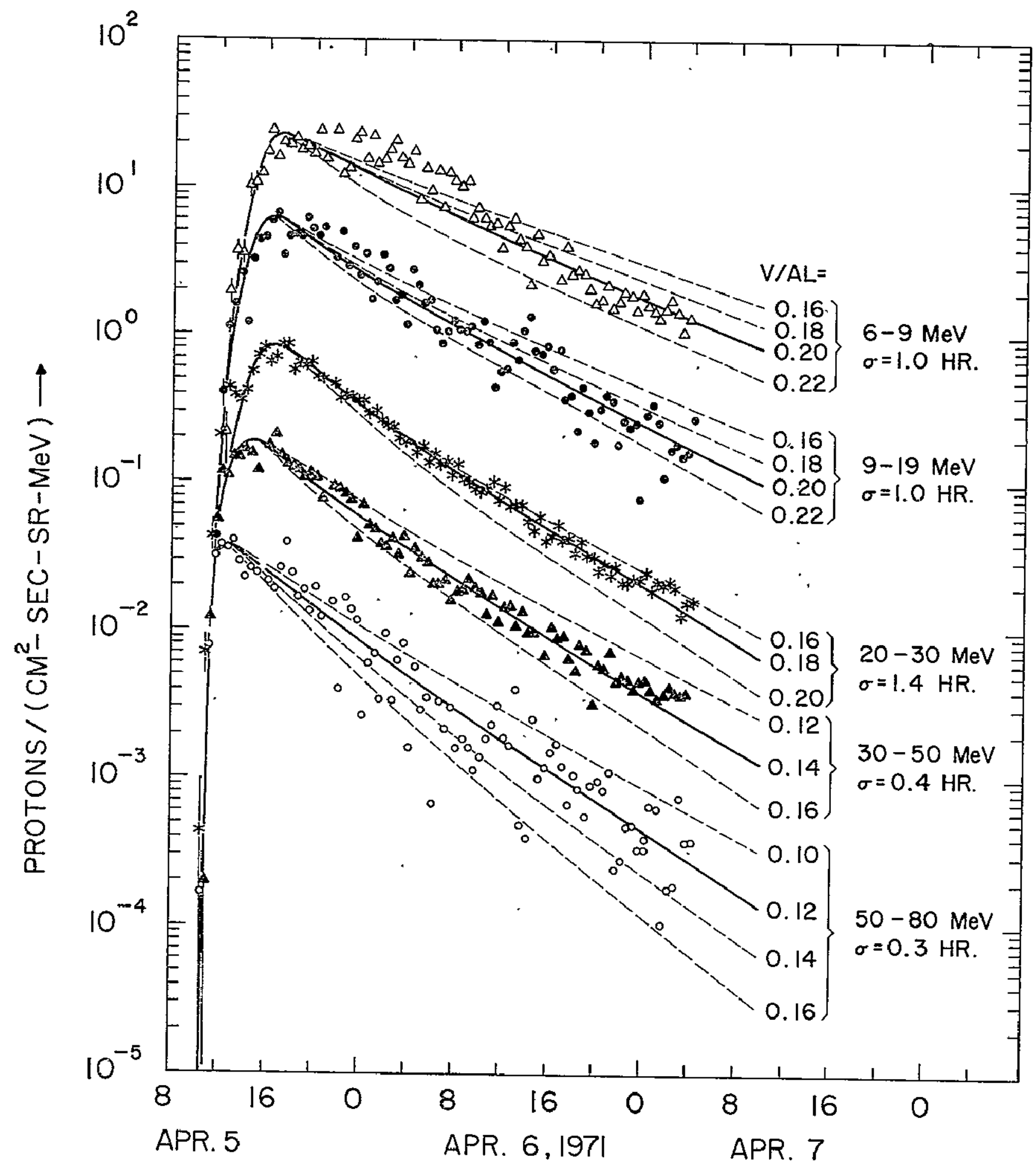

Figure 5 


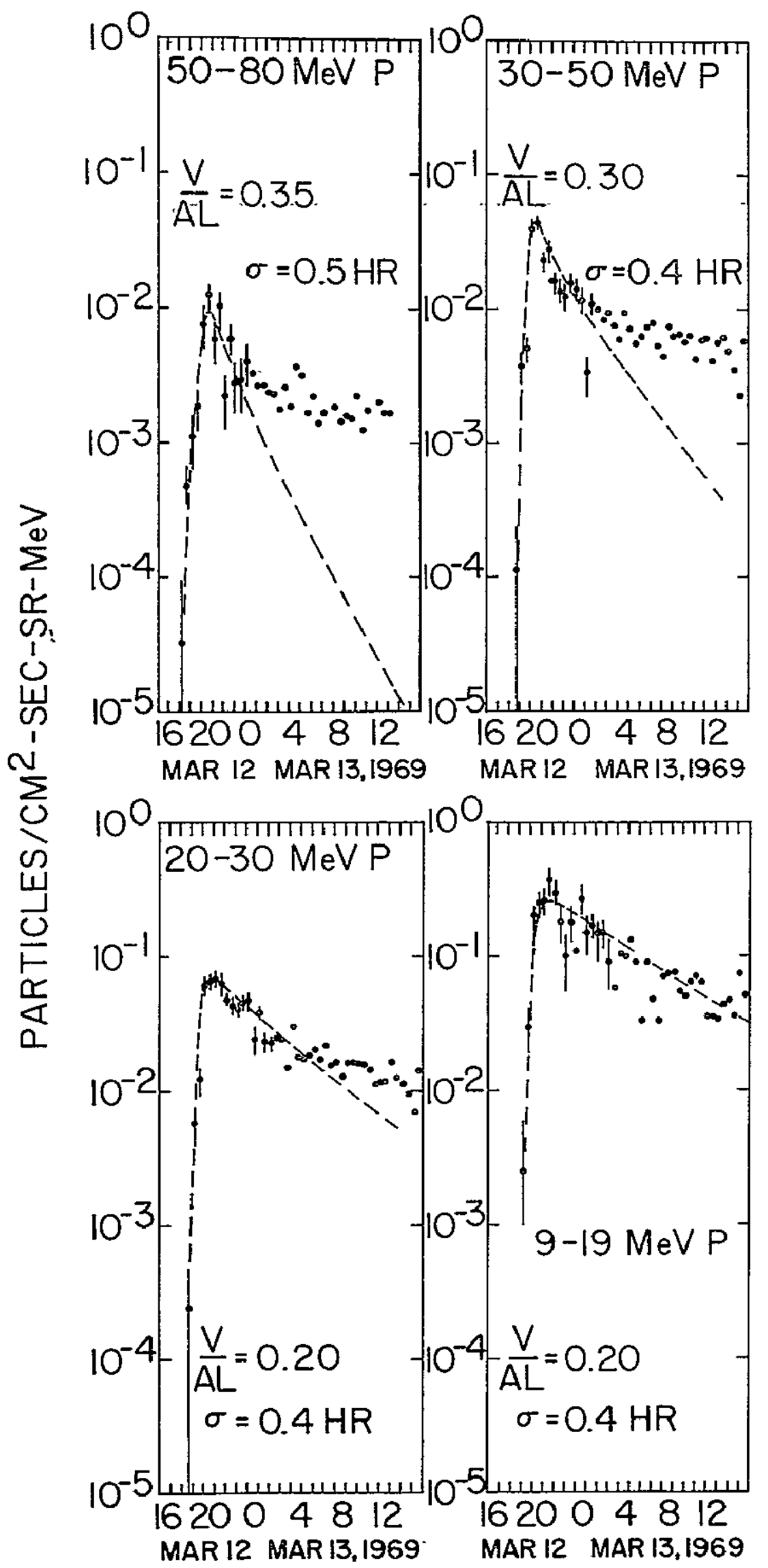

Figure 6 
FREQUENCY DISTRIBUTION OF (V/AL)

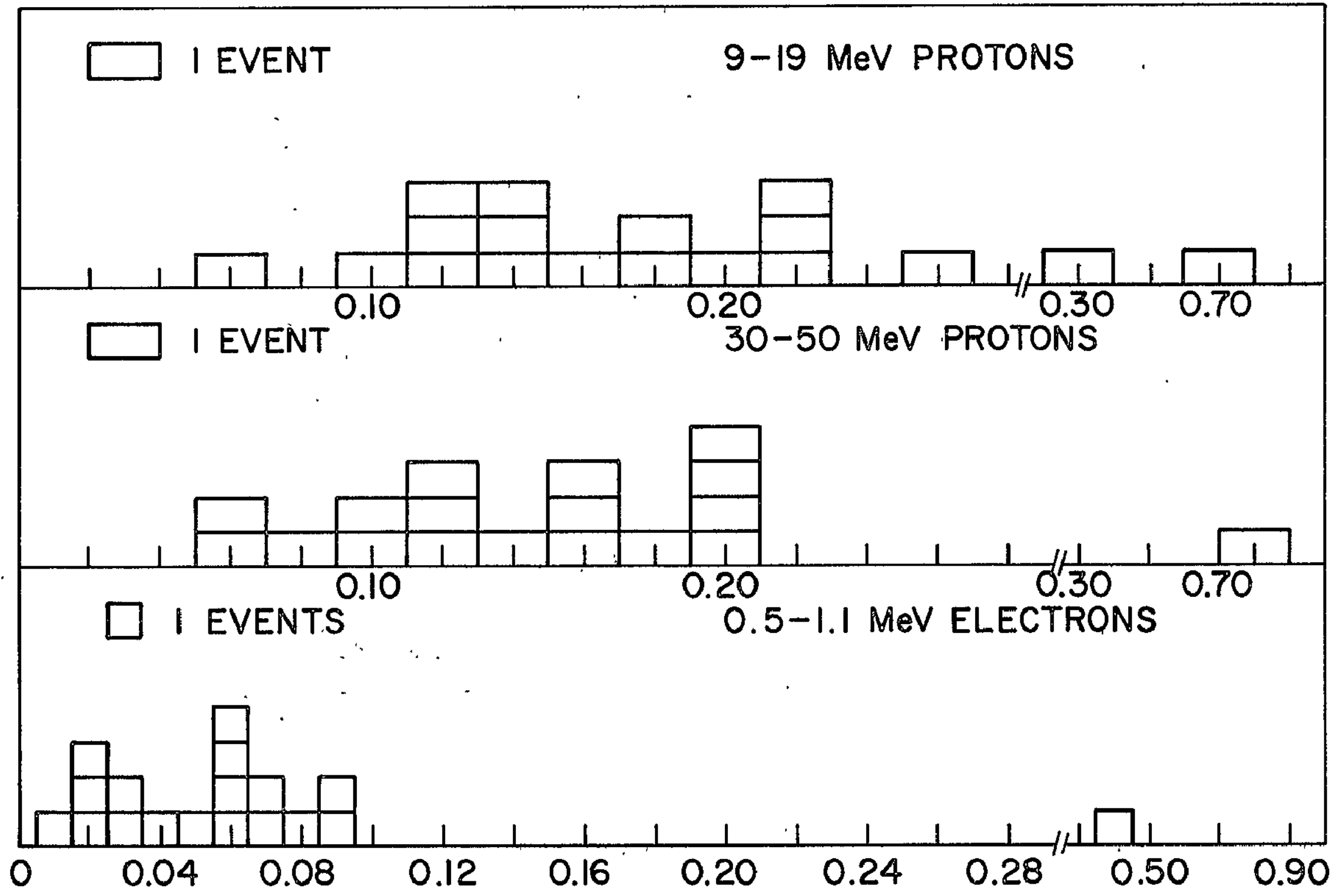

Figure 7 


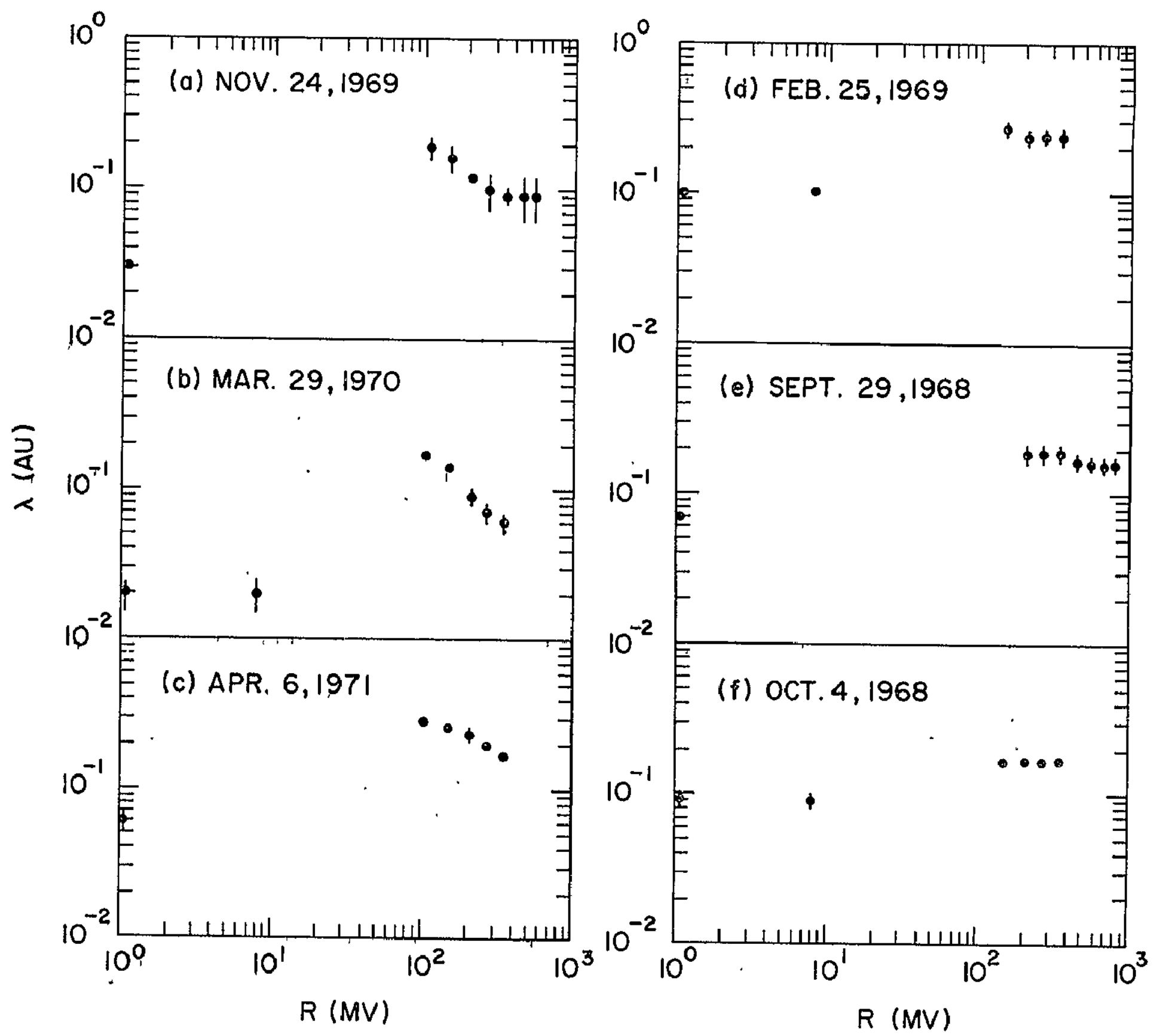

Figure 8 


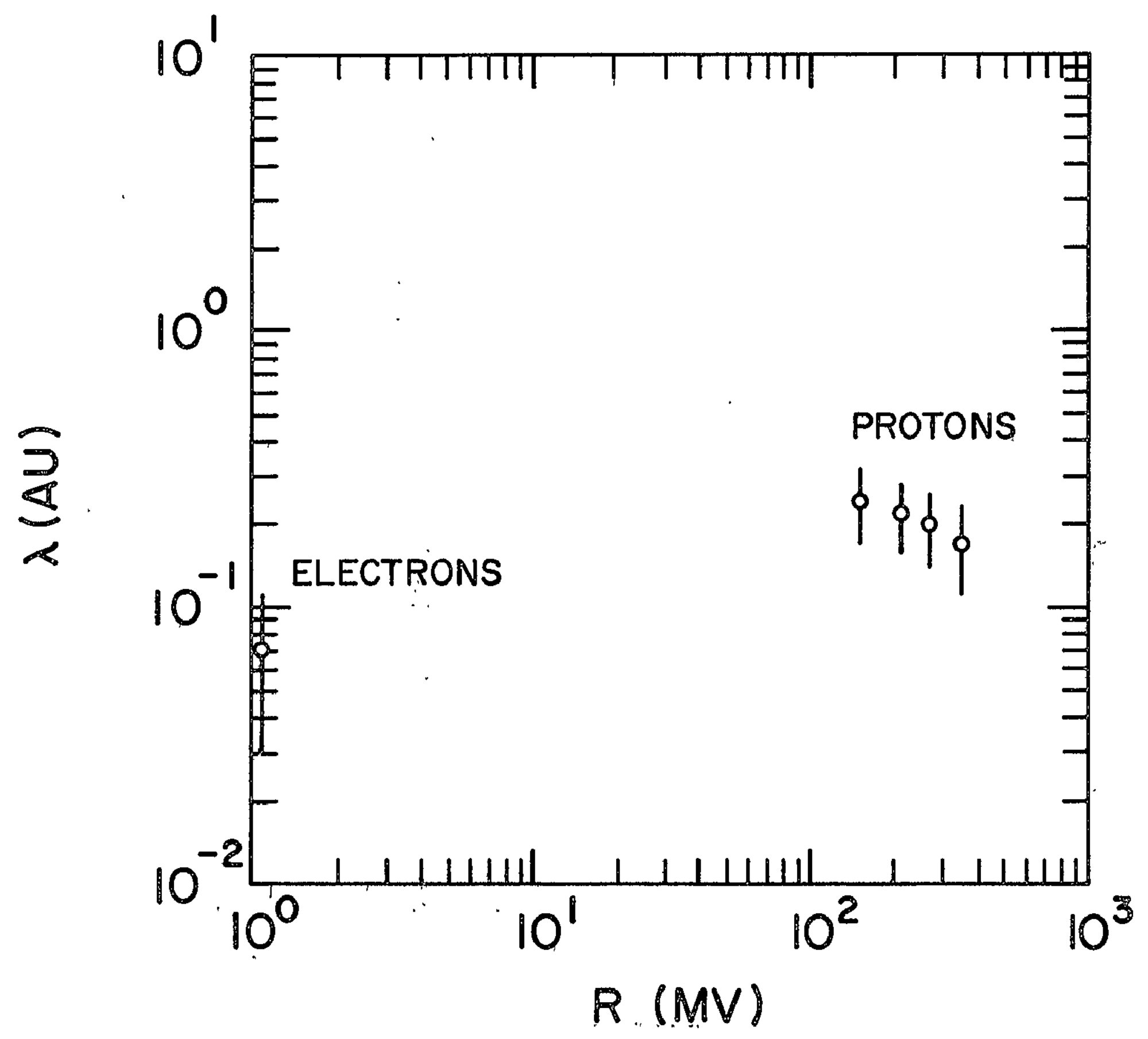

Figure 9 
FREQUENCY BISTRTBUTHON OF $\sigma$ (HOURS)

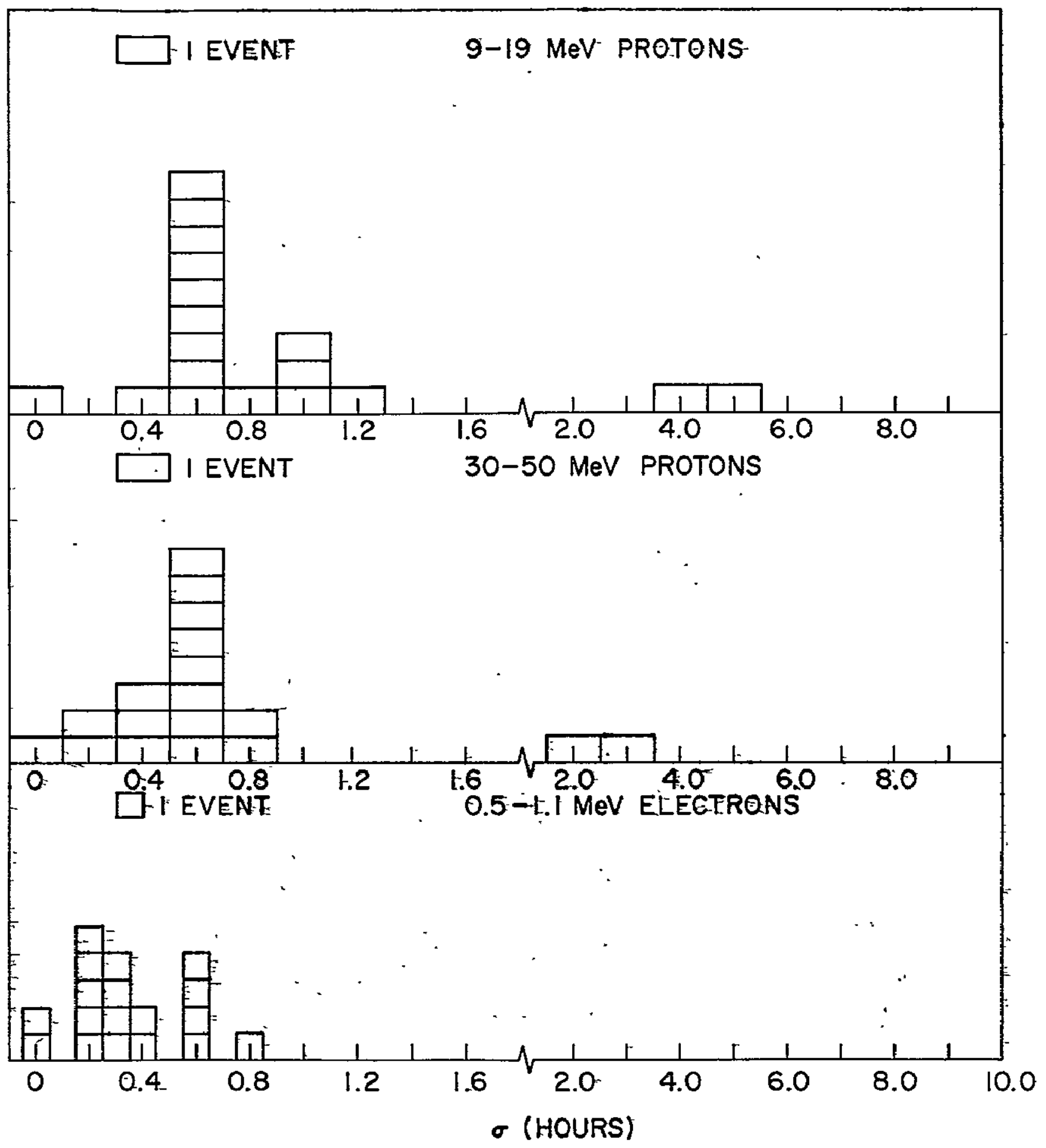

Figure 10 


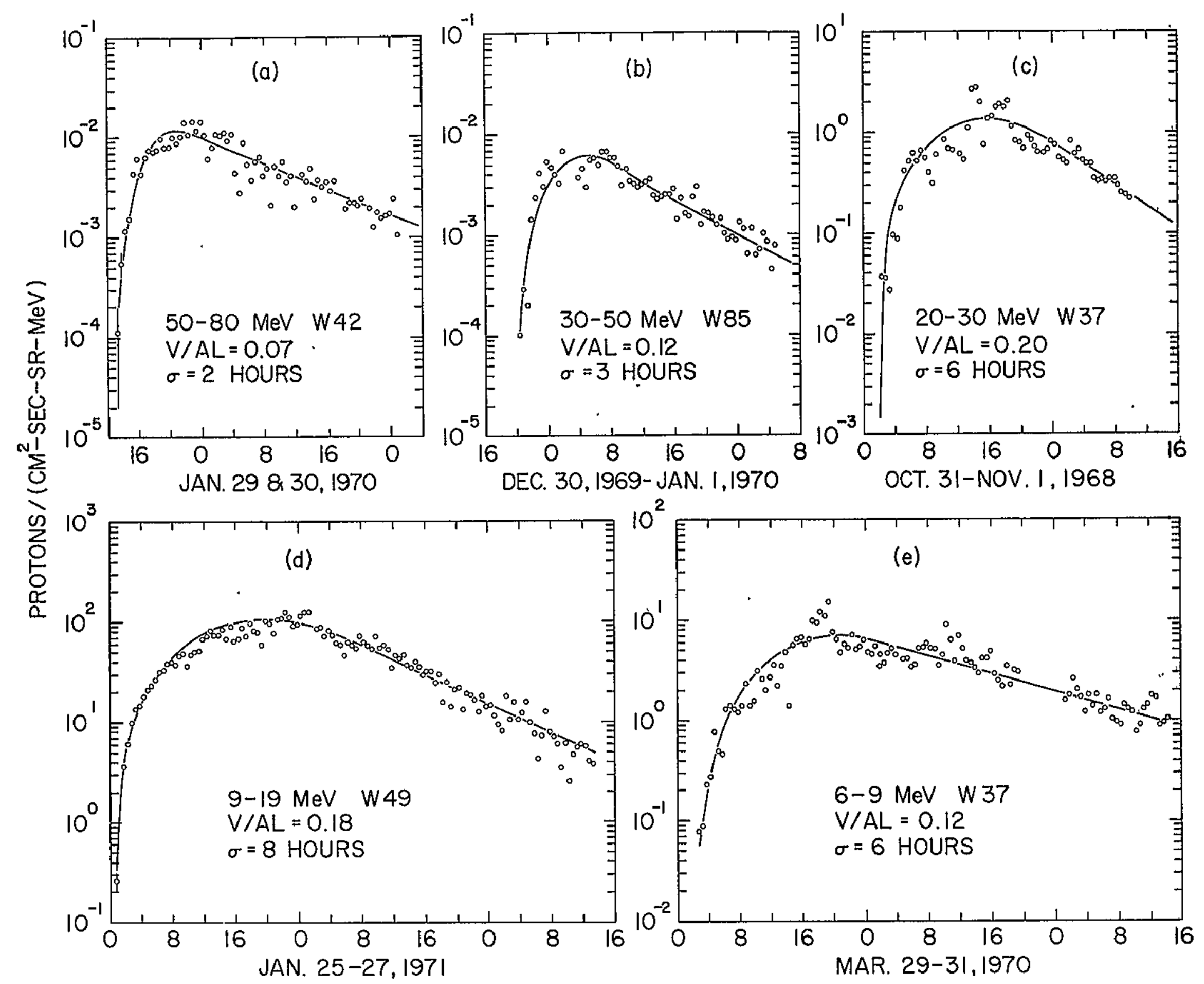

Figure 11 


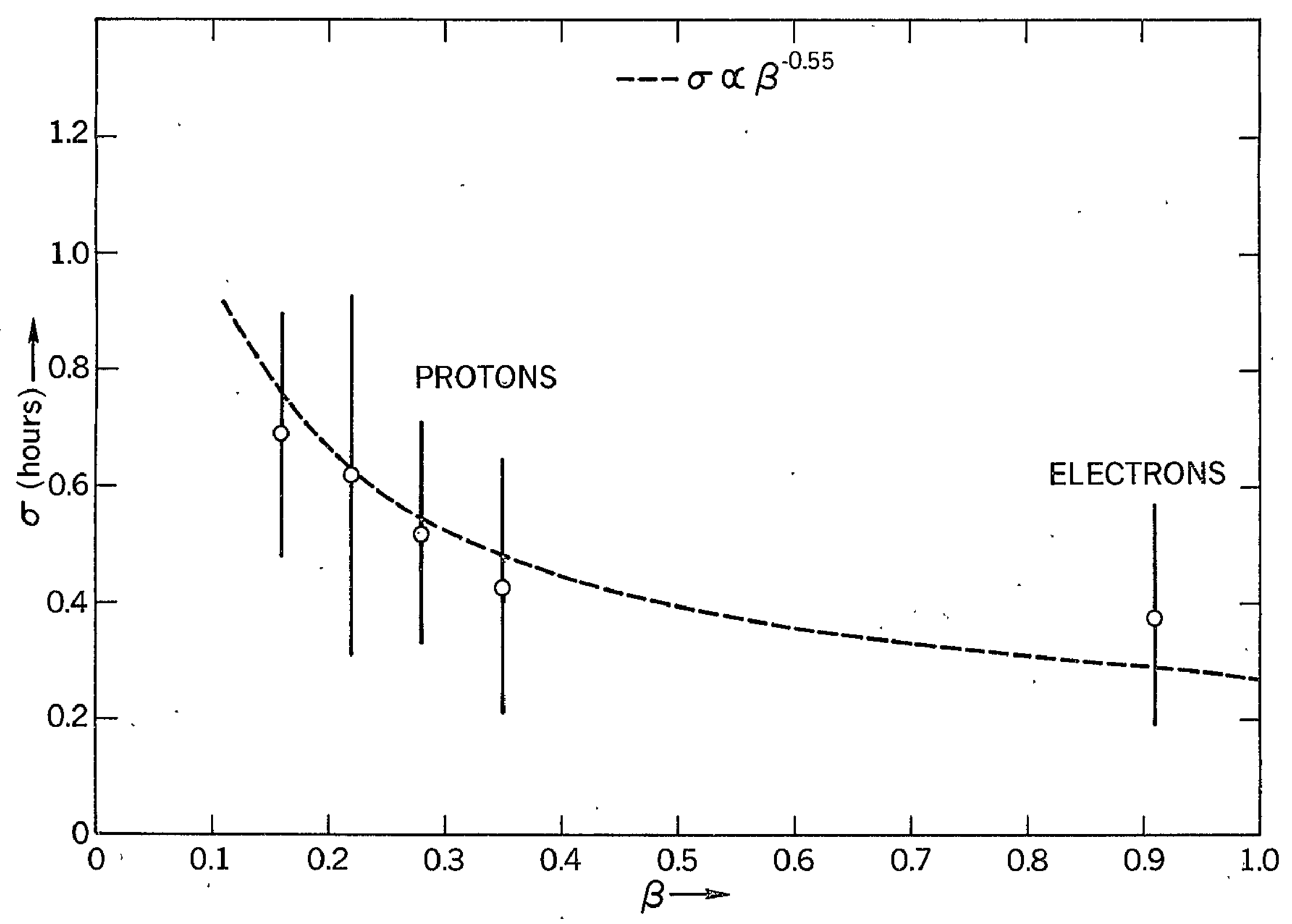

Fiqure 12 


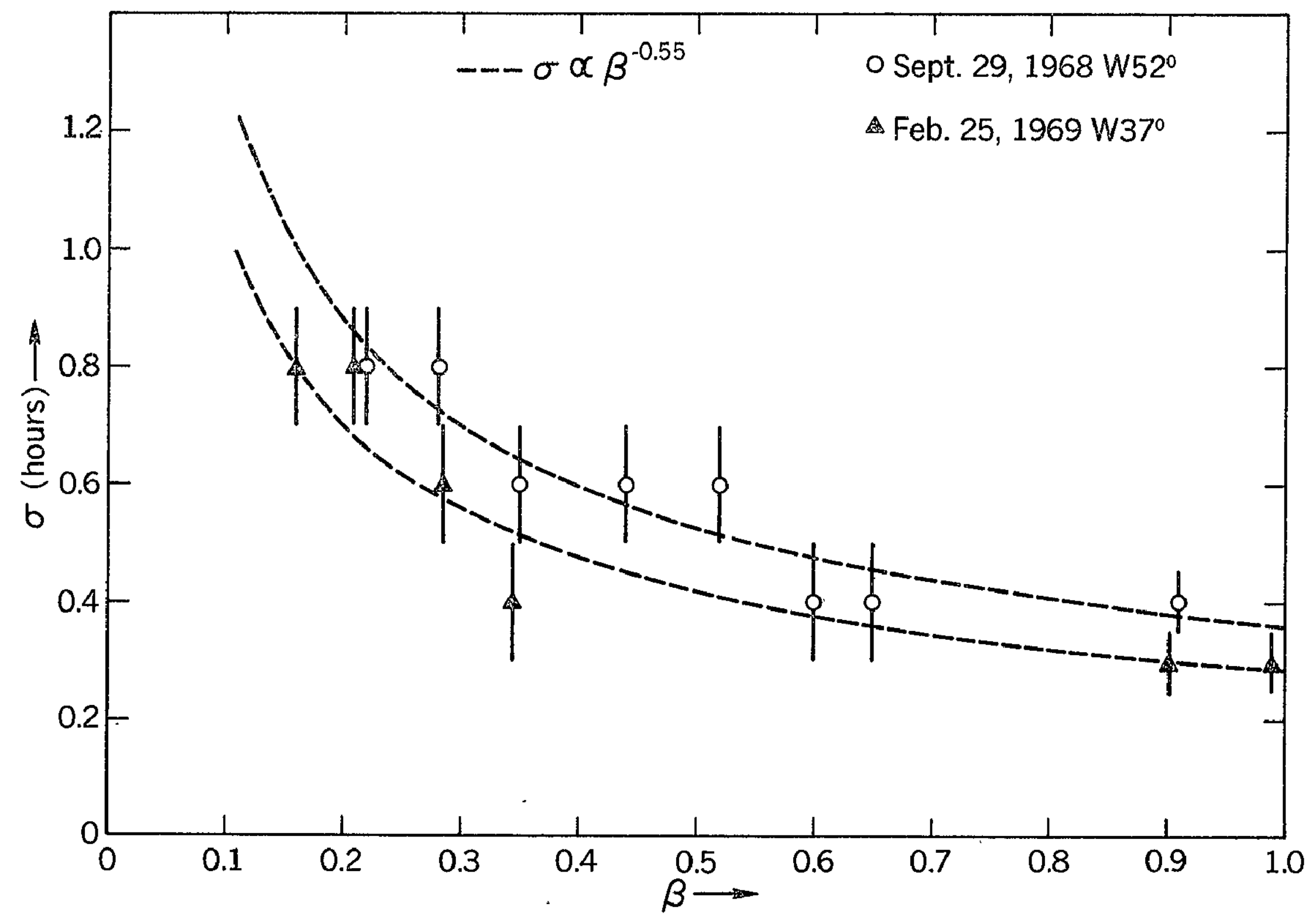

Figure 13 


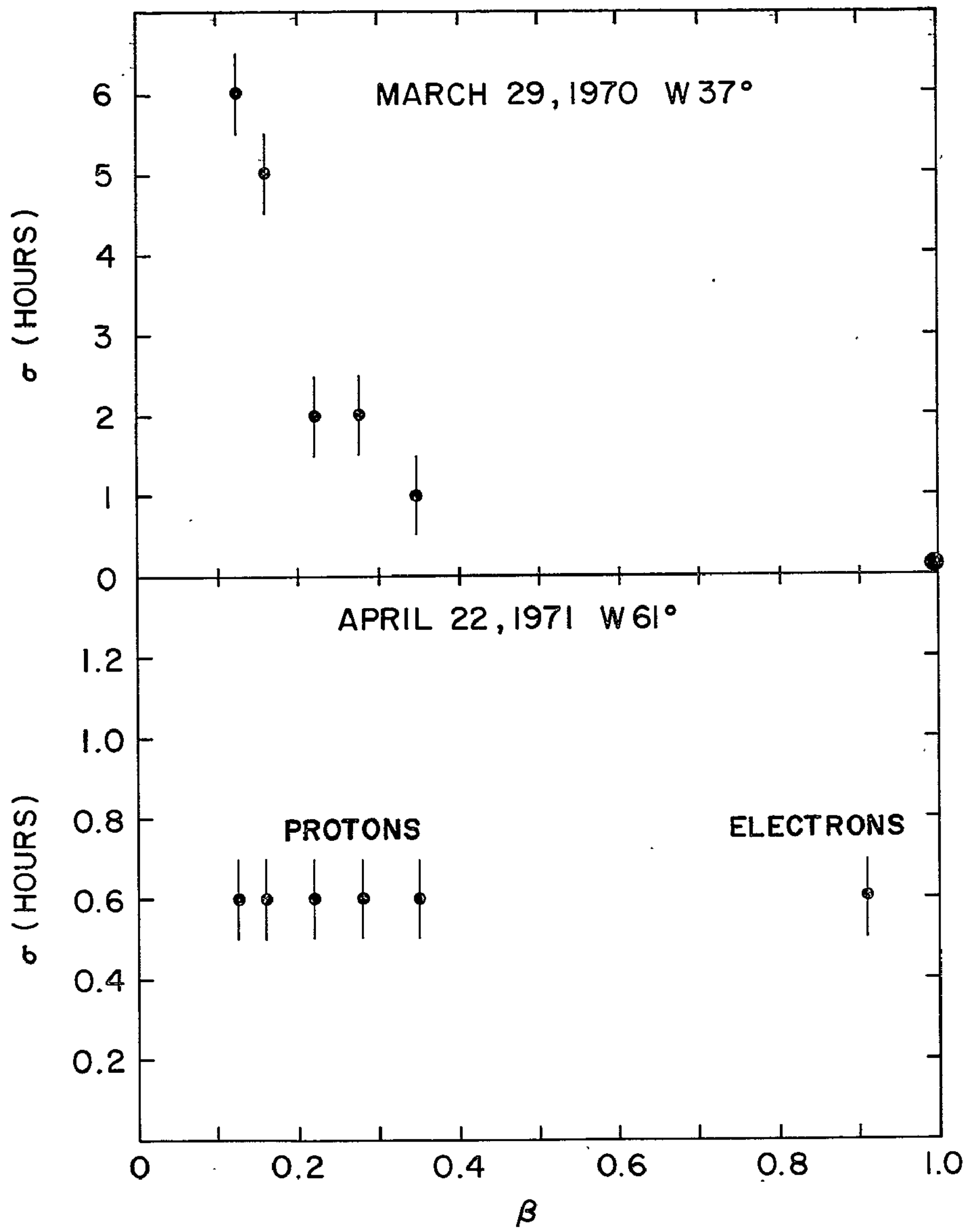

Figure 14 
BIBLIOGRAPHIC DATA SHEET

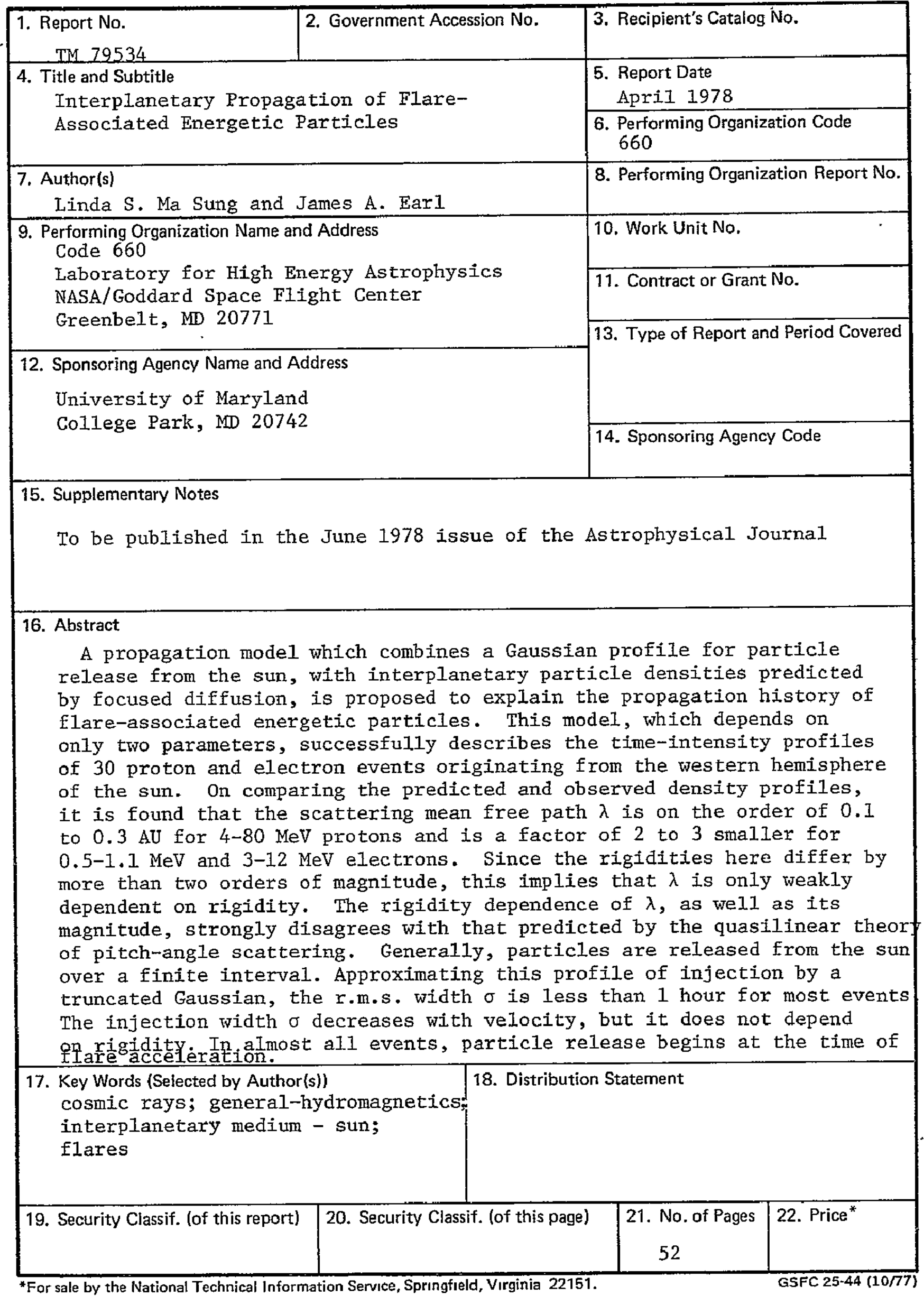

